\title{
INVARIANT TORI FOR THE BILLIARD BALL MAP
}

\author{
VALERY KOVACHEV AND GEORGI POPOV
}

\begin{abstract}
For an $n$-dimensional domain $\Omega(n \geq 3)$ with a smooth boundary which is strictly convex in a neighborhood of an elliptic closed geodesic (9), the existence of a family of invariant tori for the billiard ball map with a positive measure is proved under the assumptions of nondegeneracy and $\mathrm{N}$ elementarity, $N \geq 5$, of the corresponding to $\mathscr{O}$ Poincare map. Moreover, the conjugating diffeomorphism constructed is symplectic. An analogous result is obtained in the case $n=2$. It is shown that the lengths of the periodic geodesics determine uniquely the invariant curves near the boundary and the billiard ball map on them up to a symplectic diffeomorphism.
\end{abstract}

\section{INTRODUCTION}

Let $\Omega$ be a strictly convex and compact domain in $\mathbb{R}^{n}, n \geq 2$, with a boundary $\partial \Omega$ of class $C^{\infty}$. In this paper, we investigate the so-called billiard ball map $B$ near the boundary $S^{*} \partial \Omega$ of the coball bundle $\Sigma=B^{*} \partial \Omega=$ $\left\{(x, \xi) \in T^{*} \partial \Omega ;|\xi| \leq 1\right\}$. The billiard ball map is an exact symplectic map in the interior of $\Sigma$ [6] which is singular on the boundary $\partial \Sigma$. The local behaviour of $B$ near $\partial \Sigma$ was described by Melrose. Using the equivalence theorem for glancing hypersurfaces proved by Melrose [15] one can introduce local symplectic coordinates $(x, \xi)$ in $T^{*} \partial \Omega$ near any point $\rho \in S^{*} \partial \Omega$ so that $B^{*} \partial \Omega=\left\{\xi_{n-1} \geq 0\right\}$ and the billiard ball map $B$ assumes the form

$$
(x, \xi) \rightarrow\left(x^{\prime}, x_{n-1}-\xi_{n-1}^{1 / 2}, \xi\right), \quad \xi_{n-1} \geq 0,
$$

where $x^{\prime}=\left(x_{1}, \ldots, x_{n-2}\right)$.

This result is of particular importance for the construction of a local parametrix in $\Omega$ for the mixed problem for the wave equation. Our goal in the present paper is to construct global symplectic coordinates near a closed curve lying in $\partial \Sigma$ in which $B$ assumes a "normal form" similar to the one described above.

First we assume that $n \geq 3$. Let $\tilde{\mathscr{O}}$ be a closed bicharacteristic in $S^{*} \partial \Omega$, i.e. a closed trajectory of the Hamiltonian vector field $X_{H}$ with Hamiltonian $H(x, \xi)=|\xi|$ for $(x, \xi) \in T^{*} \partial \Omega$. Denote by $\mathscr{O} \subset \partial \Omega$ the projection of $\widetilde{\mathscr{O}}$ on $\partial \Omega$ which turns out to be a closed geodesic on $\partial \Omega$.

Received by the editors April 7, 1988.

1980 Mathematics Subject Classification (1985 Revision). Primary 58G25.

The authors were partially supported by the Bulgarian Ministry of Culture. Science, and Education under Contract 52/1988. 
We suppose that $\mathscr{O}$ is elliptic and the corresponding to $\widetilde{\mathscr{O}}$ Poincare map $P$ is nondegenerate and $N$-elementary, $N \geq 5$. Our aim is to prove the existence of a family of invariant with respect to $B$ submanifolds $\Lambda_{I} \subset \Sigma$ near $\widetilde{\mathscr{O}}$, diffeomorphic to the $(n-1)$-dimensional torus $\mathbb{T}^{n-1}=\mathbb{R}^{n-1} /(2 \pi \mathbb{Z})^{n-1}$, which are enumerated by $I$ belonging to a Cantor set $E$ with a positive Lebesgue measure in $\mathbb{R}^{n-1}$. We shall construct a smooth function $K$ in $\mathbb{R}^{n-1}, K(0)=0$, $\operatorname{grad} K(0) \neq 0$ and an exact symplectic transformation $U$ from a neighbourhood of $\widetilde{\mathscr{O}}$ into $T^{*}\left(\mathbb{T}^{n-1}\right)$ mapping $\Lambda_{I}$ into $\mathbb{T}^{n-1} \times\{I\}$ for $I \in E$ such that $B$ assumes the following "normal form"

$$
B(\varphi, I)=\left(\varphi-\operatorname{grad}\left(\frac{2}{3}(K(I))^{3 / 2}\right), I\right)
$$

for any $\varphi \in \mathbb{T}^{n-1}$ and $I \in E$.

The existence of a family of invariant tori for the billiard ball map in the case $n=3$ was announced by Svanidze in [20]. In contrast to [20] we construct the conjugating diffeomorphism $U$ symplectic and describe precisely the singularity of $B$ near $\partial \Sigma$. The motivation for the symplecticity of $U$ comes from the microlocal analysis, it arises naturally when one tries to construct quasimodes for the Laplace operator near $\partial \Omega$ using Fourier integral operators. Indeed, the billiard ball map $B$ can be considered as a boundary map $\delta_{+}$for the pair of glancing hypersurfaces $F=T_{\partial \Omega}^{*} \mathbb{R}^{n}, G=S^{*} \mathbb{R}^{n}=\left\{(x, \xi) \in T^{*} \mathbb{R}^{n} ;|\xi|=1\right\}$. Using Theorem 1 of the present paper, one of the authors proved in [18] that $F$ and $G$ can be put together into the "normal form"

$$
F=\left\{x_{n}=0\right\}, \quad G=\left\{\xi_{n}^{2}-x_{n}-K(I)=x_{n} p\left(\varphi, I, \xi_{n}\right)\right\}
$$

via an exact symplectic transformation $\chi: T^{*} \mathbb{R}^{n} \rightarrow T^{*}\left(\mathbb{T}^{n-1} \times \mathbb{R}^{1}\right)$ near $\tilde{\mathscr{O}} \subset$ $T_{\partial \Omega}^{*} \mathbb{R}^{n}$ where $p=0$ whenever $I \in E$. This is the crucial point used in [18] for the construction of quasimodes for the Laplace operator with Dirichlet (Neumann) boundary conditions whose "frequency set" is just the union of the broken bicharacteristics passing over the invariant tori of the billiard ball map.

In the case $n=2$ Lazutkin [12] proved the existence of invariant curves $\Lambda_{\omega}$ for the billiard ball map $B$ near any of the two connected components of $\partial \Sigma=S_{+}^{*} \partial \Omega \cup S^{*} \partial \Omega, S_{ \pm}^{*} \partial \Omega=\partial \Omega \times\{ \pm 1\}$, with rotation numbers $\omega$ belonging to a Cantor set $R$ with a positive measure. Moreover, he constructed a diffeomorphism $U: \mathbb{T}^{1} \times\left(\delta_{1}, \delta_{2}\right) \rightarrow \Sigma, 0<\delta_{1}<\delta_{2}$, mapping $\mathbb{T}^{1} \times\{\omega\}, \omega \in R$, into $\Lambda_{\omega}$ and such that $B_{0}=U^{-1} B U$ is given by

$$
(y, \omega) \rightarrow(y+\omega(\bmod 2 \pi), \omega) \text { on } \mathbb{T}^{1} \times\{\omega\} .
$$

In the present paper we give a more precise symplectic version of Lazutkin's result (see Theorem 2) which provides a symplectic "normal form" of $B$ on the invariant curves in a neighbourhood of $\partial \Sigma$. As a consequence we show that the lengths of the periodic broken geodesics in $\bar{\Omega} \subset \mathbb{R}^{2}$ determine uniquely the invariant curves $\Lambda_{\omega}$ and $\left.B\right|_{\Lambda_{\omega}}$ up to a symplectic map. 
Let us expose this result in more details. With any periodic point $\rho \in \Sigma \backslash \partial \Sigma$ of the billiard ball map one can associate two integers $n, m \in \mathbb{N}$ where $n$ is the period and $m$ is the winding number normalized by $2 m \leq n$. Denote by $\Gamma(m, n)$ the set of periodic orbits $\mathfrak{g}=\left\{g_{1}, \ldots, g_{n}\right\}, B g_{j}=g_{j+1(\bmod n)}$ with a winding number $m$.

Let $L(m, n)$ be the set of the lengths of the periodic broken geodesics in $\Omega$ corresponding to the periodic orbits $\mathfrak{g} \in \Gamma(m, n)$ and denote by

$$
\mathfrak{L}(\Omega)=\bigcup_{n=1}^{\infty}\left(\bigcup_{m=1}^{[n / 2]} L(m, n) \cup\left\{n l_{0}\right\}\right)
$$

the length spectrum of $\Omega$ where $l_{0}$ is the length of $\partial \Omega$.

Let $\Omega_{1}$ and $\Omega_{2}$ be two strictly convex domains in $\mathbb{R}^{2}$. Denote by $B_{1}$ and $B_{2}$ the corresponding billiard ball maps acting in $\Sigma^{1}$ and $\Sigma^{2}$ respectively. Guillemin and Melrose conjectured in [6] that if $\mathfrak{L}\left(\Omega_{1}\right)=\mathfrak{L}\left(\Omega_{2}\right)$ and the eigenvalues of the linear parts of the Poincare maps corresponding to broken geodesics in $\bar{\Omega}_{1}$ and $\bar{\Omega}_{2}$ with one and the same length coincide, then $B_{1}$ and $B_{2}$ can be conjugated by a symplectic map.

In the present paper we give a partial answer to this question. We prove that if $L_{1}(m, n)=L_{2}(m, n)$ for $m / n<\delta, \delta>0$, there exists an exact symplectic map $\chi: \Sigma^{2} \rightarrow \Sigma^{1}$ and some sets $\Sigma_{R}^{i} \subset \Sigma^{i}, i=1,2$, of positive measure (see conditions (i), (ii) in $\S 2$ ) and consisting of invariant curves for $B_{1}$ and $B_{2}$ respectively so that

$$
\chi\left(\Sigma_{R}^{2}\right)=\Sigma_{R}^{1} \quad \text { and } \quad \chi^{*}\left(\left.B_{1}\right|_{\Sigma_{R}^{1}}\right)=\left.B_{2}\right|_{\Sigma_{R}^{2}}
$$

We turn now to an outline of the paper.

In $\S 2$ the main results are formulated. $\S 3$ has a preliminary character. Here we give some facts about the so-called approximate interpolating Hamiltonian introduced by Marvizi and Melrose [14]. This is a $C^{\infty}$ function $\zeta$ defined in a neighborhood of $\widetilde{\mathscr{O}}$ in $T^{*} \partial \Omega$ which defines $S^{*} \partial \Omega$ near $\widetilde{\mathscr{O}}$ as $\{\zeta=0\}$ and such that

$$
B(\rho)=\exp \left(-\zeta^{1 / 2} X_{\zeta}\right)(\rho)+O\left(\zeta^{\infty}\right) .
$$

$\S 4$ is devoted to the construction of a completely integrable Hamiltonian $\zeta_{0}$ close to $\zeta$ and of some "action-angle" coordinates for $\zeta_{0}$. More precisely, making use of the normal form of Birkhoff for $P$, we find some symplectic coordinates $(\varphi, I) \in \mathbb{T}^{n-1} \times \mathbb{R}^{n-1}$ in which the interpolating Hamiltonian $\zeta(\varphi, I)$ can be regarded as a perturbation of a polynomial $\zeta_{0}(I)$. The coefficients of $\zeta_{0}(I)$ depend only on the normal form of Birkhoff and the length of the closed trajectories of $X_{\zeta}$ on the orbit cylinder associated with the nondegenerate trajectory $\widetilde{\mathscr{O}}$. Now the billiard ball map can be regarded as a perturbation of $B_{0}=\exp \left(-X_{\frac{2}{3} \zeta_{0}^{3 / 2}}\right)$.

In $\S 5$ we apply the Kolmogorov-Arnold-Moser (KAM) theory to the map $B_{0}$ close to $B$. For this purpose, using some ideas of $[4,5]$, we reduce the problem 
to finding invariant tori for a suitable Hamiltonian system close to a completely integrable one. The respective Hamiltonian is nondegenerate in the interior of $\Sigma$ but it has a singularity of the form $\zeta_{0}^{3 / 2}$ on $\partial \Sigma$. To overcome this difficulty we first consider the corresponding Hamiltonian systems in some compacts a way from $\partial \Sigma$ and apply a refined version of Pöschel's theorem [19] following the dependence on the various constants (see the Appendix). Next we glue the symplectic maps obtained together using some uniqueness results about the invariant tori. In $\S 6$ we consider the case $n=2$.

\section{MAIN RESUlts}

First let us recall the definition of the billiard ball map $B: \Sigma \rightarrow \Sigma$ (cf. [6]). Denote by $\nu(x)$ the exterior normal vector at $x \in \partial \Omega$ normed by $|\nu(x)|=1$. If $\xi \in T_{x}^{*} \partial \Omega$ and $|\xi|<1$, then there exists a unique vector $e(x, \xi) \in \mathbb{R}^{n}$ such that $|e(x, \xi)|=1,\langle\nu(x), e(x, \xi)\rangle<0$ and $\langle v, \xi\rangle=\langle v, e(x, \xi)\rangle$ for any $v \in T_{x} \partial \Omega$. Here $\langle$,$\rangle denotes the scalar product in \mathbb{R}^{n}$ and $T_{x}^{*} \partial \Omega$ and $T_{x} \partial \Omega$ are identified with $\mathbb{R}^{n-1}$ via the Euclidean metric. Denote by $y$ the first point of intersection of the ray $\{x+t e(x, \xi), t>0\}$ with $\partial \Omega$. This point is unique if the hypersurface is strictly convex. Let $\eta \in T_{y}^{*} \partial \Omega$ be such that $|\eta| \leq 1$ and $\langle v, e(x, \xi)\rangle=\langle v, \eta\rangle$ for any $v \in T_{y} \partial \Omega$. For $(x, \xi) \in \Sigma \cap\{|\xi|<1\}$ we define $B(x, \xi)=(y, \eta)$ and extend $B$ to $\partial \Sigma$ by $B(x, \xi)=(x, \xi)$ for $|\xi|=1$.

Let us give a precise formulation of the assumptions imposed on the closed geodesic $\mathscr{O}$ on the $C^{\infty}$ hypersurface $\partial \Omega$. Let $\rho$ be an arbitrary point of $\widetilde{\mathscr{Q}}$ and let $W \subset \partial \Sigma$ be a local transversal section of $\widetilde{\mathscr{O}}$ at $\rho$ of dimension $2 n-4$. Denote by $P$ the Poincaré map associated with the Hamiltonian flow of $X_{H}$, $H(x, \xi)=|\xi|,(x, \xi) \in T^{*} \partial \Omega$ and by $d P$ the differential of $P$ at $\rho$. First we assume that $\mathscr{O}$ is elliptic, i.e. all the eigenvalues $\lambda_{k}, \lambda_{k}^{-1}, k=1,2, \ldots, n-2$, of $d P$ are on the unit circle $\{\lambda \in \mathbb{C} ;|\lambda|=1\}$ and $\lambda_{k} \neq \pm 1$. Next we suppose that the Poincare map $P$ is $2 N+1$-elementary for some half-integer $N \geq 3 / 2$. This means that the eigenvalues of $d P$ are distinct and

$$
\prod_{k=1}^{n-2} \lambda_{k}^{j_{k}} \neq 1 \quad \text { if } 1 \leq \sum_{k=1}^{n-2}\left|j_{k}\right| \leq 2 N+1 \text {. }
$$

Let $\omega$ be the canonic symplectic form on $T^{*} \partial \Omega$. There exist local coordinates $(z, \zeta)$ mapping a neighbourhood of $\rho$ in $W$ into a neighbourhood of $O \in \mathbb{R}^{2 n-4}$ such that $\left.\omega\right|_{W}=\sum_{j=1}^{n-2} d z_{j} \wedge d \zeta_{j}$ and if we introduce polar coordinates $(\varphi, r)$ by

$$
z_{j}=\sqrt{2 r_{j}} \cos \varphi_{j}, \quad \zeta_{j}=\sqrt{2 r_{j}} \sin \varphi_{j}, \quad j=1,2, \ldots, n-2,
$$

then we can represent the Poincaré map $P: W \rightarrow W$ as

$$
P(\varphi, r)=\left(\varphi+B_{0}+B_{1} r+\cdots+O\left(|r|^{N}\right), r+O\left(|r|^{N+1}\right)\right) .
$$

Form (2.2) is called normal form of Birkhoff (cf. [9]). We make the nondegeneracy assumption

$$
\operatorname{det} B_{1} \neq 0
$$


In order to formulate our main results we shall need some additional notions.

A $C^{1}$ diffeomorphism from $T^{*} M$ into $T^{*} N$ where $M$ and $N$ are $C^{\infty}$ manifolds is said to be exact symplectic if it preserves the integrals of the fundamental 1-form over the fundamental cycles.

A function $S(y, \xi)$ is said to generate an exact symplectic transformation $T: T^{*} M \rightarrow T^{*} M$, where $M$ is either $\mathbb{R}^{n}$ or $\mathbb{T}^{n}$ if

$$
\text { graph } T=\left\{(x, \xi ; T(x, \xi)) ;(x, \xi) \in T^{*} M\right\}
$$

has the form

$$
\text { graph } T=\left\{\left(y-S_{\xi}(y, \xi), \xi ; y, \xi-S_{y}(y, \xi)\right)\right\}
$$

and $\left|\operatorname{det} S_{y \xi}(y, \xi)\right|<1$ for $(y, \xi) \in T^{*} M$.

Denote by $\Gamma \subset \mathbb{R}^{n-1}$ a set of the form

$$
\begin{aligned}
\Gamma=\left\{I \in \mathbb{R}^{n-1} ; C_{1} I_{1} \leq I_{j} \leq C_{2} I_{1}, j=2, \ldots, n-2,\right. \\
\left.\qquad C_{3} I_{1}^{2 b} \leq t_{0}-I_{n-1} \leq C_{4} I_{1}^{2 b}, 0<I_{1}<a_{0}\right\}
\end{aligned}
$$

where $C_{j}, a_{0}$ and $b<1 / 2$ are some positive constants and $2 \pi t_{0}$ is the period of $\tilde{\mathscr{O}}$.

For any point $\left(p, q, \varphi_{n-1}, I_{n-1}\right) \in \mathbb{R}^{n-2} \times \mathbb{R}^{n-2} \times T^{1} \times \mathbb{R}^{1}$ we introduce symplectic polar coordinates $(\varphi, I) \in \mathbb{T}^{n-1} \times \mathbb{R}^{n-1}$ by

$$
p_{j}=\sqrt{2 I_{j}} \cos \varphi_{j}, \quad q_{j}=\sqrt{2 I_{j}} \sin \varphi_{j}, \quad j=1, \ldots, n-2 .
$$

Denote by $V$ the set of points $\left(p, q, \varphi_{n-1}, I_{n-1}\right)$ with symplectic polar coordinates $(\varphi, I) \in \mathrm{A}^{n-1}=\mathbb{T}^{n-1} \times \Gamma$ and let $\widetilde{\mathscr{O}}^{0}=\left\{\left(0,0, \varphi_{n-1}, t_{0}\right) ; \varphi_{n-1} \in \mathbb{T}^{1}\right\} \subset$ $\bar{V}, \bar{V}$ being the closure of $V$.

Our main result is

Theorem 1. Let $\Omega$ be strictly convex in a neighbourhood of a closed elliptic geodesic $\mathscr{O} \subset \partial \Omega$ such that the corresponding Poincaré map $P$ satisfies (2.1) with $N \geq 2$ and (2.3). Then there exists an exact symplectic diffeomorphism $U: \bar{V} \rightarrow U(\bar{V}) \subset \Sigma, U\left(\widetilde{\mathscr{O}}^{0}\right)=\widetilde{\mathscr{O}}=U(\bar{V}) \cap \partial \Sigma$, a Cantor set $E \subset \bar{\Gamma}$ with a positive Lebesgue measure, $\left(0, t_{0}\right) \in E$, some smooth functions $K$ and $g$ in $\bar{\Gamma}$ and $\overline{A^{n-1}}$ respectively, $K\left(0, t_{0}\right)=0, \operatorname{grad} K\left(0, t_{0}\right) \neq 0$, so that in polar coordinates $(\varphi, I) \in \mathrm{A}^{n-1}$ the exact symplectic map $B_{0}(\varphi, I)=U^{-1} B U(\varphi, I)$ is generated by the function $-\frac{2}{3}(K(I))^{3 / 2}+g(\varphi, I)$ and

$$
g(\varphi, I)=0 \text { for any } \varphi \in \mathbb{T}^{n-1}, I \in E .
$$

Here $K \in C^{\infty}(\Gamma) \cap C^{\kappa}(\bar{\Gamma}), D_{\varphi}^{\alpha} g \in C^{\infty}\left(\mathrm{A}^{n-1}\right) \cap C^{\kappa}\left(\overline{\mathrm{A}^{n-1}}\right)$ for any multi-index $\alpha$ and $U \in C^{\infty}(V) \cap C^{\kappa-1}(\bar{V})$ where $\kappa$ is the entire part of $N-1$. Moreover, if $N \geq 3$, the set $E$ can be chosen so that

$$
\operatorname{mes}\left(E \cap B_{a}\right) / \operatorname{mes}\left(\Gamma \cap B_{a}\right)=1-O\left(a^{b}\right)
$$

where $B_{a}$ is the ball of radius $a \leq a_{0}$. 
Remark 2.1. Equality (2.5) yields $D_{\varphi}^{\alpha} D_{I}^{\beta} g(\varphi, I)=0$ for any $\varphi \in \mathbb{T}^{n-1}, I \in E$ since $E$ has no isolated points. Thus $(2.5)$ is equivalent to

$$
B_{0}(\varphi, I)=(\varphi+\operatorname{grad} \tau(I)(\bmod 2 \pi), I) \text { for }(\varphi, I) \in \mathbb{T}^{n-1} \times E
$$

where $\tau(I)=-\frac{2}{3}(K(I))^{3 / 2}$. Moreover, $D_{I}^{\beta} g(\varphi, 0)=0$ for any $\beta,|\beta| \leq \kappa$.

Let us turn now to the case $n=2$.

Suppose that $\Omega$ is a strictly convex domain in $\mathbb{R}^{2}$ with a $C^{\infty}$ smooth boundary $\partial \Omega$ of length $l_{0}=2 \pi \mathfrak{l}$. Denote $\Gamma=\left[\mathfrak{l}-\delta_{0}, \mathfrak{l}\right] \subset \mathbb{R}^{1}, A=\mathbb{T}^{1} \times \Gamma$ for some positive constant $\delta_{0}$. Then the following theorem is valid which is a counterpart of Theorem 1 for $n=2$.

Theorem 2. Let $\Omega$ be a strictly convex planar domain with a smooth boundary of length $l_{0}$. Then there exists an exact symplectic diffeomorphism $U: A \rightarrow \Sigma$, $U\left(\mathbb{T}^{1} \times\{\mathfrak{l}\}\right)=S_{+}^{*} \partial \Omega$, a Cantor set $E \subset \Gamma$ with a positive Lebesgue measure, $\mathfrak{l} \in E$, and some $C^{\infty}$ functions $K(I)$ and $g(\varphi, I)$ in $\Gamma$ and $\mathrm{A}$ respectively, $K(\mathfrak{l})=0, K^{\prime}(\mathfrak{l})<0$, such that the exact symplectic map $B_{0}=U^{-1} B U$ is generated in $A$ by the function $-\frac{2}{3}(K(I))^{3 / 2}+g(\varphi, I)$ and (2.5) is satisfied for any $\varphi \in \mathbb{T}^{1}, I \in E$.

Moreover, the set $E$ can be chosen so that for any $\delta \in\left(0, \delta_{0}\right]$

$$
\delta-\operatorname{mes}(E \cap[\mathfrak{l}-\delta, \mathfrak{l}]) \leq C_{n} \delta^{N}
$$

Remark 2.2. Equality (2.5) for $n=2$ yields again

$$
B_{0}(\varphi, I)=\left(\varphi+\tau^{\prime}(I), I\right)+Q(\varphi, I)
$$

where $\tau(I)=-\frac{2}{3}(K(I))^{3 / 2}$ and $Q(\varphi, I)=0$ for any $(\varphi, I) \in \mathbb{T}^{1} \times E$.

Remark 2.3. The invariant tori given in Theorem 2 can be enumerated by their rotation numbers which in the two-dimensional case determine the invariant tori uniquely. The set $R$ of rotation numbers is defined by the small denominator condition as follows. First for any $j \in \mathbb{N}$ consider

$$
\begin{aligned}
& R_{j}=\left\{\omega \in\left(4^{-j}, 4^{-j+1}\right) ;\left|\omega k_{1}-k_{2}\right| \geq \mu 2^{-j^{2}-2 j}\left|k_{1}\right|^{-\sigma}\right. \\
& \left.\quad \text { for any }\left(k_{1}, k_{2}\right) \in \mathbb{Z}^{2} \backslash\{0\}\right\}
\end{aligned}
$$

where $\sigma>1$ and $\mu>0$ do not depend on $j$. Now, we write $R=\left(\bigcup_{j=1}^{\infty} R_{j}\right) \cap$ $\left(0, \delta_{0}\right)$ for some $\delta_{0}>0$. Then the set $E$ can be given by $E=\left\{I \in \Gamma ; \tau^{\prime}(I) \in \bar{R}\right\}$ as we shall see in the proof of Theorem 2 .

Denote $\Sigma_{R}=U\left(\mathbb{T}^{1} \times E\right)$. Obviously, $\Sigma_{R}$ is a subset of $\Sigma$ such that

(i) $\Sigma_{R}$ is the union of invariant curves of $B$ with rotation numbers in $R$.

(ii) $\operatorname{mes}\left(\Sigma^{\delta}\right)-\operatorname{mes}\left(\Sigma^{\delta} \cap \Sigma_{R}\right) \leq C_{N} \delta^{N}$ for any $\delta \in\left(0, \delta_{0}\right], N>0$ and some $C_{N}>0$ where $\Sigma^{\delta}=\partial \Omega \times[1-\delta, 1]$.

Let $\Omega_{1}$ and $\Omega_{2}$ be two strictly convex domains in $\mathbb{R}^{2}$. Denote by $B_{1}$ and $B_{2}$ the corresponding billiard ball maps acting in $\Sigma^{1}$ and $\Sigma^{2}$ respectively and 
by $L_{i}(m, n)$ the sets $L(m, n)$ defined as in $\S 1$ for $\Omega_{i}, i=1,2$. Let $R$ be given as in Remark 2.3, and $\Sigma_{R}^{i}, i=1,2$, be the union of the invariant curves of $B_{i}, i=1,2$, with rotation numbers in $R$.

Theorem 3. Suppose that $L_{1}(m, n)=L_{2}(m, n)$ if $m / n<\delta$ for some $\delta>0$. Then there exists an exact symplectic map $\chi: \Sigma^{2} \rightarrow \Sigma^{1}$ such that $\chi\left(\Sigma_{R}^{2}\right)=\Sigma_{R}^{1}$ and $\chi^{*}\left(\left.B_{1}\right|_{\Sigma_{R}^{1}}\right)=\left.B_{2}\right|_{\Sigma_{R}^{2}}$. Moreover, the set $E$ and the restriction of $K$ on $E$ are determined uniquely by the set of rotation numbers $R$ and by $L(m, n)$ for $m / n<\delta$.

Theorem 1 was announced in a slightly weaker form in [11] and Theorems 2 and 3 in [17] where an idea of the proof was also given.

\section{INTERPOLATING HAMILTONIAN}

The equivalence theorem of Melrose [15] for nondegenerate glancing points (cf. also [8]) can be applied to the transversally intersecting hypersurfaces $S^{*} \mathbb{R}^{n}$

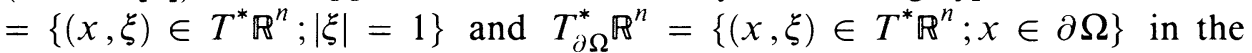
symplectic manifold $T^{*} \mathbb{R}^{n}$ since the manifold of glancing points $\partial \Sigma=S^{*} \partial \Omega$ consists entirely of nondegenerate glancing points provided that $\Omega$ is strictly convex in a neighbourhood of $\mathscr{O}$ (see [13]). Thus in a neighbourhood $U \subset$ $T^{*} \partial \Omega$ of any point $(x, \xi) \in \widetilde{\mathscr{O}} \subset \partial \Sigma$ we can introduce symplectic coordinates $(y, \eta)=\left(y^{\prime}, y_{n-1}, \eta^{\prime}, \eta_{n-1}\right)=\chi(x, \xi)$ such that $\partial \Sigma \cap U=\left\{\eta_{n-1}=0\right\}, \eta_{n-1} \geq$ 0 in $\Sigma \cap U$ and

$$
\chi \circ B \circ \chi^{-1}(y, \eta)=\left(y^{\prime}, y_{n-1}-\eta_{n-1}^{1 / 2}, \eta\right) .
$$

Then

$$
B(x, \xi)=\exp \left(-\eta_{n-1}^{1 / 2}(x, \xi) X_{\eta_{n-1}}\right)(x, \xi) .
$$

We can say that $B$ is locally interpolated by the Hamiltonian flow generated by the function $\eta_{n-1}(x, \xi)$ which is called a local interpolating Hamiltonian for the billiard ball map.

We shall use the following proposition whose assertion is given in [13] without proof.

Proposition 3.1. Let $\zeta_{j}, j=1,2$, be local interpolating Hamiltonians for the billiard ball map in a neighbourhood $U$ of $\rho_{0} \in \partial \Sigma$. Then $\zeta_{1}-\zeta_{2}$ vanishes to infinite order on $\partial \Sigma$.

Proof. As noted in [13], the assertion of Proposition 3.1 can be derived from the proof of Melrose [15]. For the sake of completeness here we give a direct proof of it which is close to the proof in the two-dimensional case [14].

Let $(x, \xi)$ and $(y, \eta)$ be symplectic coordinates in a neighbourhood $U$ of $\rho_{0} \in \partial \Sigma$ such that $x\left(\rho_{0}\right)=\xi\left(\rho_{0}\right)=y\left(\rho_{0}\right)=\eta\left(\rho_{0}\right)=0, U \cap \partial \Sigma=\left\{\xi_{n-1}=0\right\}=$ $\left\{\eta_{n-1}=0\right\}, \xi_{n-1} \geq 0$ and $\eta_{n-1} \geq 0$ in $U \cap \Sigma$ and

$$
B(x, \xi)=\left(x^{\prime}, x_{n-1}-\xi_{n-1}^{1 / 2}, \xi\right), \quad B(y, \eta)=\left(y^{\prime}, y_{n-1}-\eta_{n-1}^{1 / 2}, \eta\right)
$$


in $U \cap \Sigma$. Here $\xi_{n-1}=\zeta_{1}, \eta_{n-1}=\zeta_{2}$ are the respective local interpolating Hamiltonians. Denote by $x=x(y, \eta), \xi=\xi(y, \eta)$ the symplectic change of variables $(y, \eta) \rightarrow(x, \xi)$ defined in a neighbourhood $U_{0}$ of $(0,0)$. From (3.1) we have

$$
\begin{gathered}
\xi_{j}(y, \eta)=\xi_{j}\left(y^{\prime}, y_{n-1}-\eta_{n-1}^{1 / 2}, \eta\right), \quad j=1, \ldots, n-1, \\
x_{j}(y, \eta)=x_{j}\left(y^{\prime}, y_{n-1}-\eta_{n-1}^{1 / 2}, \eta\right), \quad j=1, \ldots, n-2, \\
x_{n-1}(y, \eta)-\xi_{n-1}^{1 / 2}(y, \eta)=x_{n-1}\left(y^{\prime}, y_{n-1}-\eta_{n-1}^{1 / 2}, \eta\right)
\end{gathered}
$$

in $U_{0} \cap\left\{\eta_{n-1} \geq 0\right\}$ if $U_{0}$ is small enough.

Now we shall make use of the following lemma.

Lemma 3.2. Let $p(z, \zeta)$ be a $C^{\infty}$ function which satisfies for all $\zeta \geq 0$ the equality

$$
p\left(z-\zeta^{1 / 2}, \zeta\right)=p(z, \zeta)+O\left(\zeta^{\infty}\right)
$$

Then we have $(\partial p / \partial z)(z, \zeta)=O\left(\zeta^{\infty}\right)$.

To prove the lemma, it suffices to show that

$$
\frac{\partial^{n+1} p}{\partial z \partial \zeta^{n}}(z, 0)=0
$$

for any nonnegative integer $n$. This equality is proved by induction using Taylor's series of both sides of $(3.3)$ around $(z, 0)$.

From the first $2 n-3$ equalities of (3.2) we obtain

$$
\begin{array}{ll}
\left\{\eta_{n-1}, \xi_{j}\right\}=\frac{\partial \xi_{j}}{\partial y_{n-1}}(y, \eta)=O\left(\eta_{n-1}^{\infty}\right), & j=1, \ldots, n-1, \\
\left\{\eta_{n-1}, x_{j}\right\}=\frac{\partial x_{j}}{\partial y_{n-1}}(y, \eta)=O\left(\eta_{n-1}^{\infty}\right), & j=1, \ldots, n-2,
\end{array}
$$

because $\xi_{j}(y, \eta), j=1, \ldots, n-1$, and $x_{j}(y, \eta), j=1, \ldots, n-2$, are smooth functions of $\left(y_{n-1}, \eta_{n-1}\right)$ depending on the parameters $\left(y^{\prime}, \eta^{\prime}\right)$ and we can apply Lemma 3.2. Here $\{$,$\} stands for Poisson's bracket in T^{*} \mathbb{R}^{n-1}$ associated with the standard symplectic form.

If we consider, conversely, $\eta_{n-1}$ as a function of $(x, \xi)$, we find

$$
\begin{array}{ll}
\frac{\partial \eta_{n-1}}{\partial x_{j}}=-\left\{\eta_{n-1}, \xi_{j}\right\}=O\left(\eta_{n-1}^{\infty}\right), & j=1, \ldots, n-1, \\
\frac{\partial \eta_{n-1}}{\partial \xi_{j}}=\left\{\eta_{n-1}, x_{j}\right\}=O\left(\eta_{n-1}^{\infty}\right), & j=1, \ldots, n-2,
\end{array}
$$

hence $\eta_{n-1}=\eta_{n-1}\left(\xi_{n-1}\right)+O\left(\xi_{n-1}^{\infty}\right)$ and $\partial \eta_{n-1}(0) / \partial \xi_{n-1}>0$ since the change is nonsingular and in $U \cap \Sigma$ both $\eta_{n-1}$ and $\xi_{n-1}$ are nonnegative. Thus $\xi_{n-1}=$ 
$f\left(\eta_{n-1}\right)+O\left(\eta_{n-1}^{\infty}\right)$ where $f$ is a smooth function such that $f(0)=0, f^{\prime}(0)>0$ and $f\left(\eta_{n-1}\right)>0$ for $\eta_{n-1}>0$. From the last equality of (3.2) we have

$$
x_{n-1}(y, \eta)-f^{1 / 2}\left(\eta_{n-1}\right)=x_{n-1}\left(y^{\prime}, y_{n-1}-\eta_{n-1}^{1 / 2}, \eta\right)+O\left(\eta_{n-1}^{\infty}\right),
$$

whence we obtain

$$
\frac{\partial x_{n-1}}{\partial y_{n-1}}(y, \eta)=\frac{\partial x_{n-1}}{\partial y_{n-1}}\left(y^{\prime}, y_{n-1}-\eta_{n-1}^{1 / 2}, \eta\right)+O\left(\eta_{n-1}^{\infty}\right) .
$$

Now Lemma 3.2 yields

$$
\frac{\partial^{2} x_{n-1}}{\partial^{2} y_{n-1}}(y, \eta)=O\left(\eta_{n-1}^{\infty}\right),
$$

hence

$$
x_{n-1}(y, \eta)=a\left(y^{\prime}, \eta\right) y_{n-1}+b\left(y^{\prime}, \eta\right)+O\left(\eta_{n-1}^{\infty}\right)
$$

where $a\left(y^{\prime}, \eta\right)$ and $b\left(y^{\prime}, \eta\right)$ are $C^{\infty}$ functions. Then from (3.4) we obtain $a\left(y^{\prime}, \eta\right)=\left(f\left(\eta_{n-1}\right) / \eta_{n-1}\right)^{1 / 2}+O\left(\eta_{n-1}^{\infty}\right)$ for $\eta_{n-1}>0$. Now, as in [14] we have

$$
\begin{aligned}
1 & =\left\{\xi_{n-1}, x_{n-1}\right\}=\left\{f\left(\eta_{n-1}\right),\left(f\left(\eta_{n-1}\right) / \eta_{n-1}\right)^{1 / 2} y_{n-1}+b\left(y^{\prime}, \eta\right)\right\}+O\left(\eta_{n-1}^{\infty}\right) \\
& =f^{\prime}\left(\eta_{n-1}\right)\left(f\left(\eta_{n-1}\right) / \eta_{n-1}\right)^{1 / 2}+O\left(\eta_{n-1}^{\infty}\right) \text { for } \eta_{n-1}>0,
\end{aligned}
$$

hence

$$
f^{3 / 2}\left(\eta_{n-1}\right)=\eta_{n-1}^{3 / 2}+C+O\left(\eta_{n-1}^{\infty}\right) .
$$

Since $f(0)=0$, then $C=0$, i.e. $\xi_{n-1}=\eta_{n-1}+O\left(\eta_{n-1}^{\infty}\right)$ and Proposition 3.1 is proved.

Let $\left\{\varphi_{j}\right\}_{j=1}^{J}$ be a smooth partition of unity in a neighbourhood $U$ of $\tilde{\mathscr{O}}$ in $T^{*} \partial \Omega$ and let $\zeta_{j}$ be local interpolating Hamiltonians for the billiard ball map defined respectively in a neighbourhood of $\operatorname{supp} \varphi_{j}$. Now define $\zeta=$ $\sum_{j=1}^{J} \varphi_{j} \zeta_{j}$. From Proposition 3.1 it follows that $\zeta-\zeta_{j}$ vanishes to infinite order on $\partial \Sigma$. Now we have $B(\rho)=\exp \left(-\zeta^{1 / 2} X_{\zeta}\right)(\rho)+O\left(\zeta^{\infty}(\rho)\right)$ in a neighbourhood of $\tilde{\mathscr{O}}$. Moreover, $\zeta=0$ defines $\partial \Sigma$ in a neighbourhood of $\tilde{\mathscr{O}}$ and the bicharacteristic lines passing through the points $\rho \in \partial \Sigma$ coincide with $\left\{\exp \left(t X_{\zeta}\right)(\rho) ; \zeta=0, t \in \mathbb{R}^{1}\right\}$.

The function $\zeta$ is called an approximate interpolating Hamiltonian. In the case $n=2$ its construction is carried out directly in [14] and its uniqueness in the sense of Proposition 3.1 is proved.

\section{Construction of SUITABle "ACTION-ANGLE" COORDinAtes}

4.1. Let $S$ be a transversal to $\widetilde{\mathscr{O}}$ in $\Sigma$ and $S_{h}=\{\rho \in S ; \zeta(\rho)=h\}, S_{0}=W$. Since 1 is not an eigenvalue of the differential $d P$ of the Poincare map $P$ at $\rho \in S_{0} \cap \widetilde{\mathscr{O}}$, there exists a smooth family of closed trajectories $\widetilde{\mathscr{O}}_{h}$ of $X_{\zeta}$ contained respectively in the sets $\{\rho \in \Sigma ; \zeta(\rho)=h\}$ for $|h|$ small which form an orbit cylinder and $\widetilde{\mathscr{O}}_{0}=\widetilde{\mathscr{O}}$.

We shall use the normal form of Birkhoff for the Poincaré map $P_{\zeta}: S_{\zeta} \rightarrow S_{\zeta}$ depending smoothly on the parameter $\zeta$. 
Proposition 4.1. Let the Poincaré map be $2 N+1$-elementary, $N \geq 3 / 2$. Then there exist symplectic coordinates $(p, q, \zeta, t)$ in a neighbourhood of $S$, i.e. $\omega=$ $\sum_{j=1}^{n-2} d p_{j} \wedge d q_{j}+d \zeta \wedge d t$ such that $S \subset\{t=0\}$ and if we denote $P_{\zeta}(p, q)=$ $\left(p^{*}, q^{*}\right)$, then

$$
\begin{aligned}
p_{j}^{*} & =p_{j} \cos \Phi_{j}\left(r^{\prime}, \zeta\right)-q_{j} \sin \Phi_{j}\left(r^{\prime}, \zeta\right)+f_{j}(p, q, \zeta), \\
q_{j}^{*} & =p_{j} \sin \Phi_{j}\left(r^{\prime}, \zeta\right)+q_{j} \cos \Phi_{j}\left(r^{\prime}, \zeta\right)+f_{n-2+j}(p, q, \zeta), \\
j & =1, \ldots, n-2,
\end{aligned}
$$

where $r^{\prime}=\left(r_{1}, \ldots, r_{n-2}\right), r_{j}=\left(p_{j}^{2}+q_{j}^{2}\right) / 2$ and

$$
\left|f_{j}(p, q, \zeta)\right| \leq C\left(|p|^{2}+|q|^{2}\right)^{N+1 / 2}, \quad j=1, \ldots, 2 n-4 .
$$

Moreover,

$$
\Phi_{j}\left(r^{\prime}, \zeta\right)=\frac{\partial A}{\partial r_{j}}\left(r^{\prime}, \zeta\right), \quad j=1, \ldots, n-2,
$$

where $A$ is a polynomial of $r^{\prime}$ of degree at most $N$ with $C^{\infty}$ coefficients and $\rho_{0}^{h}(0,0, h)$ lie on the cylinder of orbits of $X_{\zeta}$. We choose $A\left(r^{\prime}, \zeta\right)$ so that $A(0, \zeta)=0$.

The proof of Proposition 4.1 is analogous to that given in $[9,16]$, constructing a generating function of the respective canonical transformation depending smoothly on $\zeta$. Thus we construct the coordinates $p$ and $q$ in $S$. Then we extend $p$ and $q$ to a neighbourhood of $S$ so that $X_{\zeta} p_{j}=X_{\zeta} q_{j}=0$, $j=1, \ldots, n-2$, and supplement $p, q, \zeta$ to a symplectic basis in a neighbourhood of $S$. It is easily seen that in this basis

$$
X_{\zeta}=\partial / \partial t
$$

Let $2 \pi t_{0}$ be a period of $\widetilde{\mathscr{O}}$. Denote by $\widetilde{G}: U_{0} \rightarrow \widetilde{G}\left(U_{0}\right) \subset \mathbb{R}^{2 n-2}$ the symplectic map $\widetilde{G}(\rho)=\left(p, q, t / t_{0}, t_{0} \zeta\right)$ defined in a neighbourhood $U_{0}$ of $S$ and set $G^{0}=\left.\widetilde{G}\right|_{S_{\zeta}}$.

For $\delta$ small enough we denote

$$
\begin{aligned}
V_{\delta}=\left\{\left(p, q, \varphi_{n-1}, I_{n-1}\right) \in \mathbb{R}^{n-2} \times \mathbb{R}^{n-2} \times \mathbb{T}^{1} \times\left[t_{0}-\delta, t_{0}+\delta\right]\right. & \\
& \left.p_{j}^{2}+q_{j}^{2} \leq 2 \delta, j=1, \ldots, n-2\right\}
\end{aligned}
$$

with a symplectic form $\tilde{\omega}=d p \wedge d q+d I_{n-1} \wedge d \varphi_{n-1}$. We shall denote by

$$
(\varphi, I)=\pi\left(p, q, \varphi_{n-1}, I_{n-1}\right) \in \Pi^{n-1}=\mathbb{T}^{n-1} \times(0, \delta]^{n-2} \times\left[t_{0}-\delta, t_{0}+\delta\right]
$$

the respective polar coordinates in $V_{\delta}$ determined by

$$
p_{j}=\sqrt{2 I_{j}} \cos \varphi_{j}, \quad q_{j}=\sqrt{2 I_{j}} \sin \varphi_{j}, \quad j=1, \ldots, n-2 .
$$

Note that for $\tilde{\omega}^{0}=\sum_{j=1}^{n-1} d I_{j} \wedge d \varphi_{j}$ we have $\pi^{*} \tilde{\omega}^{0}=\tilde{\omega}$ in $\Pi^{n-1}$. 
4.2. Let $l(\zeta)$ be the period of periodic trajectory $\widetilde{\mathscr{O}}_{\zeta}$ from the cylinder of orbits, thus $l(0)=2 \pi t_{0}$. Denote by $l^{0}(\zeta)$ and $A^{0}\left(r^{\prime}, \zeta\right)$ Taylor's expansions of $l(\zeta)$ and $A(r, \zeta)$ with respect to $\zeta$ at $\zeta=0$ up to degrees $M$ and $M+1$ respectively, $M$ sufficiently large. Let $\zeta_{0}$ be the solution of the equation

$$
2 \pi t_{0}-\int_{0}^{\zeta} l^{0}(h) d h-A^{0}\left(r^{\prime}, \zeta\right)=2 \pi I_{n-1}, \quad \zeta\left(0, t_{0}\right)=0,
$$

for $\left(r^{\prime}, t_{0}-I_{n-1}\right) \in[-\delta, \delta]^{n-1}$. Then we have

$$
\frac{\partial \zeta_{0}\left(0, t_{0}\right)}{\partial I_{n-1}}<0
$$

The following proposition provides "action-angle" coordinates for a completely integrable Hamiltonian $\zeta_{0}$ which is close to $\zeta$.

Proposition 4.2. For $\delta$ small enough there exists a neighbourhood $U_{\delta}$ of $\widetilde{\mathscr{O}}$ in $T^{*} \partial \Omega$ and an exact symplectic transformation $G: V_{\delta} \rightarrow U_{\delta}$ of class $C^{\infty}$ such that $G^{*} \zeta=\zeta \circ G$ has the form

$$
G^{*} \zeta\left(p, q, \varphi_{n-1}, I_{n-1}\right)=\zeta_{0}(I)+\tilde{\zeta}\left(p, q, \varphi_{n-1}, I_{n-1}\right)
$$

and

$$
\left|\tilde{\zeta}\left(p, q, \varphi_{n-1}, I_{n-1}\right)\right| \leq C\left(\left|I^{\prime}\right|^{N+1 / 2}+\left|\zeta_{0}\right|^{M+2}\right)
$$

where $C$ does not depend on $\delta, I^{\prime}=\left(I_{1}, \ldots, I_{n-2}\right), I_{j}=\left(p_{j}^{2}+q_{j}^{2}\right) / 2, j=$ $1, \ldots, n-2, I=\left(I^{\prime}, I_{n-1}\right)$.

Proof. First we shall construct suitable smooth coordinates in a neighbourhood $U$ of $\widetilde{\mathscr{O}}$.

Denote by $g^{t}$ the Hamilton flow of $r_{n-1}=t_{0} \zeta$ and for $\rho \in S_{\zeta}, \rho$ in a neighbourhood of $\rho_{0}^{0}=\tilde{\mathscr{O}} \cap S_{0}$ denote by $\tau(\rho)$ the smallest positive time such that $g^{\tau(\rho)}(\rho) \in S_{\zeta}, \tau\left(\rho_{0}^{0}\right)=2 \pi$. Let $s(\rho)$ be the smallest nonnegative time for which $g^{-s(\rho)}(\rho) \in S$ for $\rho$ in a neighbourhood of $\widetilde{\mathscr{O}}$.

Before constructing a suitable coordinate system near $\widetilde{\mathscr{O}}$ it is convenient to represent the map $P_{\zeta}$ in the form $P_{\zeta}=P^{0}+P^{1}$ (for the sake of brevity we drop $\zeta)$ where $P^{0}(p, q)=(\tilde{p}, \tilde{q})$,

$$
\begin{gathered}
\tilde{p}_{j}=p_{j} \cos \Phi_{j}\left(r^{\prime}, \zeta\right)-q_{j} \sin \Phi_{j}\left(r^{\prime}, \zeta\right), \\
\tilde{q}_{j}=p_{j} \sin \Phi_{j}\left(r^{\prime}, \zeta\right)+q_{j} \cos \Phi_{j}\left(r^{\prime}, \zeta\right), \quad j=1, \ldots, n-2, \\
P^{1}(p, q)=\left(f_{1}(p, q, \zeta), \ldots, f_{2 n-4}(p, q, \zeta)\right) .
\end{gathered}
$$

Obviously, $P^{1}(\rho)=O\left(\left|r^{\prime}(\rho)\right|^{N+1 / 2}\right)$.

Let $\chi \in C^{\infty}\left(\mathbb{R}^{1}\right), 0 \leq \chi \leq 1, \chi^{\prime}(t) \geq 0$ and $\chi(t)=0$ in $(-\infty, \varepsilon), \chi(t)=1$ in $(2 \pi-\varepsilon, \infty)$ for some $\varepsilon>0$ small enough. Denote by $P_{t}^{0}: \mathbb{R}^{2 n-4} \rightarrow \mathbb{R}^{2 n-4}$ the map $P_{t}^{0}(p, q)=\left(p^{*}, q^{*}\right)$ where

$$
p_{j}^{*}=p_{j} \cos \left(\chi(t) \Phi_{j}\left(r^{\prime}, \zeta\right)\right)-q_{j} \sin \left(\chi(t) \Phi_{j}\left(r^{\prime}, \zeta\right)\right),
$$


$q_{j}^{*}=p_{j} \sin \left(\chi(t) \Phi_{j}\left(r^{\prime}, \zeta\right)\right)+q_{j} \cos \left(\chi(t) \Phi_{j}\left(r^{\prime}, \zeta\right)\right), \quad j=1, \ldots, n-2$.

Denote by $P_{t}^{1}$ the map $P_{t}^{1}(\rho)=\chi(t) P^{1}(\rho)$ and set $P^{t}=P_{t}^{0}+P_{t}^{1}$. Now consider the map

$$
F^{\prime}(\rho)=(p(\rho), q(\rho))=P^{s(\rho)} \circ G^{0} \circ g^{-s(\rho)}(\rho) .
$$

It is easy to see that $F^{\prime}$ is a well-defined $C^{\infty}$ map in a neighbourhood of $\widetilde{\mathscr{Q}}$ since $P^{t}=\mathrm{Id}$ for $t \leq \varepsilon$ and $P^{t}=P_{\zeta}$ for $t \geq 2 \pi-\varepsilon$.

Next we define the smooth function $\varphi_{n-1}$ as follows. Denote $T(t, \rho)$ $=t+\chi(t)(2 \pi-\tau(\rho))$. We set for $\rho \in U$ a neighbourhood of $\widetilde{\mathscr{O}}, \varphi_{n-1}(\rho)=$ $T\left(s(\rho), g^{-s(\rho)}(\rho)\right)$.

It is easy to see that $\varphi_{n-1}: U \rightarrow \mathbb{T}^{1}$ is a well-defined $C^{\infty}$ function. Moreover, the map

$$
U \ni \rho \rightarrow F(\rho)=\left(F^{\prime}(\rho), \varphi_{n-1}(\rho), r_{n-1}(\rho)\right)
$$

is a diffeomorphism for $U$ small enough and $\left.F\right|_{U_{0}}=\left.\widetilde{G}\right|_{U_{0}}$.

Let $(\varphi, r)=\pi\left(p, q, \varphi_{n-1}, r_{n-1}\right)$ be the respective polar coordinates

$$
p_{j}=\sqrt{2 r_{j}} \cos \varphi_{j}, \quad q_{j}=\sqrt{2 r_{j}} \sin \varphi_{j}, \quad j=1, \ldots, n-2 .
$$

Denote $r^{\prime}=\left(r_{1}, \ldots, r_{n-2}\right)$.

Now in polar coordinates $(\varphi, r)$ we denote by $U_{1}$ the set

$$
U_{1}=\left\{\rho \in U ; C_{1} r_{1}(\rho) \leq r_{j}(\rho) \leq C_{2} r_{1}(\rho), j=2, \ldots, n-2\right\}
$$

where $0<C_{1}<1<C_{2}$ are some constants.

Lemma 4.3. In $U_{1}$ we have

$$
\begin{aligned}
& \left|\left\{r_{j}, r_{k}\right\}(\rho)\right| \leq C\left|r^{\prime}(\rho)\right|^{N+1}, \quad j, k \leq n-1, \\
& \left\{r_{j}, \varphi_{k}\right\}(\rho)=\delta_{j k}+O\left(\left|r^{\prime}\right|^{N}\right), \quad j \leq n-2, k \leq n-1, \\
& \left\{r_{n-1}, \varphi_{n-1}\right\}(\rho)=1+O\left(\left|r^{\prime}\right|\right) .
\end{aligned}
$$

Proof. From (4.1), (4.2), and (4.7) it is easy to see that

$$
\left|\left(g^{l}\right)^{*} r-r\right| \leq C\left|r^{\prime}\right|^{N+1}, \quad|t| \leq 4 \pi .
$$

Indeed,

$$
\begin{aligned}
\left(g^{t}\right)^{*} F^{\prime}(\rho) & =P^{s(\rho)+t} \circ G^{0} \circ g^{-s(\rho)}(\rho)=P^{s(\rho)+t} \circ P^{-s(\rho)} \circ F^{\prime}(\rho) \\
& =P_{0}^{s(\rho)+t} \circ P_{0}^{-s(\rho)} \circ F^{\prime}(\rho)+O\left(\left|r^{\prime}\right|^{N+1 / 2}\right)
\end{aligned}
$$

and $\left(g^{l}\right)^{*} r_{n-1}=r_{n-1}$ which imply (4.9). Moreover, from the above equality it follows that for $j, k \leq n-2$

$$
\left(g^{t}\right)^{*} \varphi_{j}(\rho)=(\chi(s(\rho)+t)-\chi(s(\rho))) \Phi_{j}(r(\rho))+O\left(\left|r^{\prime}(\rho)\right|^{N}\right) .
$$


Therefore,

$$
\begin{aligned}
X_{r_{n-1}}\left\{r_{j}, \varphi_{k}\right\} & =\left\{r_{j}, X_{r_{n-1}} \varphi_{k}\right\}+O\left(\left|r^{\prime}\right|^{N+1 / 2}\right) \\
& =\left\{r_{j}, \chi^{\prime}(s(\rho)) \Phi_{k}(r)\right\}+O\left(\left|r^{\prime}\right|^{N}\right) \\
& =\Phi_{k}(r) \chi^{\prime \prime}(s(\rho))\left\{r_{j}, s(\rho)\right\}+O\left(\left|r^{\prime}\right|^{N}\right) .
\end{aligned}
$$

On the other hand,

$$
X_{r_{n-1}}\left\{s(\rho), r_{j}\right\}=O\left(\left|r^{\prime}\right|^{N+1 / 2}\right) .
$$

Thus $\left\{s(\rho), r_{j}\right\}=O\left(\left|r^{\prime}\right|^{N+1 / 2}\right)$ for $j \leq n-2$ and

$$
\left\{r_{j}, \varphi_{k}\right\}(\rho)=\left\{r_{j}, \varphi_{k}\right\}\left(g^{-s(\rho)}(\rho)\right)+O\left(\left|r^{\prime}\right|^{N}\right)=\delta_{j k}+O\left(\left|r^{\prime}\right|^{N}\right) .
$$

Now we shall prove that

$$
\tau(\rho)=f(r(\rho))+O\left(\left|r^{\prime}\right|^{N+1 / 2}\right), \quad \rho \in S,
$$

for some smooth function $f$ such that $f(0)=2 \pi$. Let $\rho^{0} \in S, \rho=(0, r(\rho))$ in polar coordinates. Denote by $g_{j}^{t}, t \in \mathbb{R}^{1}$, the Hamiltonian flow of $r_{j}$, $j=1, \ldots, n-1$. Then $\rho_{1}=g_{n-1}^{\tau\left(\rho^{0}\right)}\left(\rho^{0}\right) \in S$ and $r\left(\rho_{1}\right)=r\left(\rho^{0}\right)+O\left(\left|r^{\prime}\right|^{N+1}\right)$. Now taking $\tau_{j}\left(\rho^{0}\right)=\Phi_{j}\left(r^{\prime}\left(\rho^{0}\right), \zeta\left(\rho^{0}\right)\right), j=1, \ldots, n-2$, from Proposition 4.1 we have

$$
g_{1}^{\tau_{1}\left(\rho^{0}\right)} \circ \cdots \circ g_{n-1}^{\tau_{n-2}\left(\rho^{0}\right)} g_{n-1}^{\tau\left(\rho^{0}\right)}\left(\rho^{0}\right)=\rho^{0}+O\left(\left|r^{\prime}\right|^{N+1 / 2}\right)
$$

On the other hand,

$$
\left[X_{r_{j}}, X_{r_{k}}\right]=X_{\left\{r_{j}, r_{k}\right\}}=O\left(\left|r^{\prime}\right|^{N+1 / 2}\right)
$$

and as in [2] it is easy to see that

$$
g_{j}^{t_{j}} \circ g_{k}^{t_{k}}(\rho)=g_{k}^{t_{k}} \circ g_{j}^{t_{j}}(\rho)+O\left(\left|r^{\prime}(\rho)\right|^{N+1 / 2}\right) .
$$

Let $\rho \in S, r(\rho)=r\left(\rho^{0}\right)$. Then $g_{1}^{t_{1}(\rho)} \circ \cdots \circ g_{n-2}^{t_{n-2}(\rho)}\left(\rho^{0}\right)=\rho$ for some $t_{j}(\rho) \in \mathbb{R}^{1}$ and in view of (4.12), (4.13) we obtain

$$
g_{1}^{\tau_{1}\left(\rho^{0}\right)} \circ \cdots \circ g_{n-2}^{\tau_{n-2}\left(\rho^{0}\right)} \circ g^{\tau\left(\rho^{0}\right)}(\rho)=\rho+O\left(\left|r^{\prime}\right|^{N+1 / 2}\right),
$$

thus $g^{\tau\left(\rho^{0}\right)}(\rho)=\rho_{1}+O\left(\left|r^{\prime}\right|^{N+1 / 2}\right)$ where

$$
\rho_{1}=g_{n-2}^{-\tau_{n-2}\left(\rho^{0}\right)} \circ \cdots \circ g_{1}^{-\tau_{1}\left(\rho^{0}\right)}(\rho) \in S
$$

or

$$
\operatorname{dist}\left(g^{\tau\left(\rho^{0}\right)}(\rho), S\right)=O\left(\left|r^{\prime}\right|^{N+1 / 2}\right) .
$$

Therefore $\tau(\rho)=\tau\left(\rho^{0}\right)+O\left(\left|r^{\prime}\right|^{N+1 / 2}\right), \rho^{0}=(0, r(\rho))$. Obviously $\tau\left(\rho^{0}\right)$ is a smooth function of $\sqrt{r_{1}}, \ldots, \sqrt{r_{n-2}}$. Let $f(r)$ be its Taylor's series up to order $2 N$. Then $f(r)$ satisfies (4.11) and $f(0)=2 \pi$. On the other hand, consider 
$\tau(\rho)$ as a function of $p, q$ and denote by $\tau^{*}(p, q)$ its Taylor series up to order $2 N$. Then

$$
\tau^{*}(p, q)=f\left(\left(p_{1}^{2}+q_{1}^{2}\right) / 2, \ldots,\left(p_{n-1}^{2}+q_{n-1}^{2}\right) / 2\right)
$$

which implies that $f$ is a polynomial of $r_{j}=\left(p_{j}^{2}+q_{j}^{2}\right) / 2, j=1, \ldots, n-2$.

The second estimate of (4.8) for $k=n-1$ follows from (4.11) which yields

$$
\varphi_{n-1}(\rho)=\tilde{T}(s(\rho), r(\rho))+O\left(\left|r^{\prime}\right|^{N+1 / 2}\right) \text {. }
$$

Here

$$
\widetilde{T}(t, r)=t+\chi(t)(2 \pi-f(r)) .
$$

Thus $X_{r_{j}} \varphi_{n-1}=O\left(\left|r^{\prime}\right|^{N}\right), j \leq n-2$.

Finally, we have $X_{r_{n-1}} \varphi_{n-1}=1+O\left(\left|r^{\prime}\right|\right)$ since $f(0)=2 \pi$.

It is easy to see that in polar coordinates $(\varphi, I)$ we have

$$
\pi_{*} F_{*} X_{r_{j}}(\rho)=\frac{\partial}{\partial \varphi_{j}}+\sum_{k=1}^{n-1}\left(c_{j k}(\rho) \frac{\partial}{\partial r_{k}}+d_{j k}(\rho) \frac{\partial}{\partial \varphi_{k}}\right), \quad j \leq n-1,
$$

where

$$
\begin{aligned}
& \left|c_{j k}(\rho)\right| \leq C\left|r^{\prime}\right|^{N+1}, \quad\left|d_{j k}(\rho)\right| \leq C\left|r^{\prime}\right|^{N}, \quad j \leq n-2, k \leq n-1, \\
& \left|c_{n-1, k}(\rho)\right| \leq C\left|r^{\prime}\right|^{N+1}, \quad k \leq n-1, \\
& \left|d_{n-1, k}(\rho)\right| \leq C, \quad k \leq n-2, \quad\left|d_{n-1, n-1}(\rho)\right| \leq C\left|r^{\prime}\right|
\end{aligned}
$$

uniformly in $U_{1}$. Indeed, we have

$$
\pi_{*} F_{*} X_{r_{j}}(\rho)=\sum_{k=1}^{n-1}\left(\left\{r_{j}, r_{k}\right\}(\rho) \frac{\partial}{\partial r_{k}}+\left\{r_{j}, \varphi_{k}\right\}(\rho) \frac{\partial}{\partial \varphi_{k}}\right)
$$

and by Lemma 4.3 we prove (4.14).

Let $c \in(0, \delta)^{n-2} \times(-\delta, \delta)$ and $M_{c}=\{r=c\}$. Then $M_{c}$ is a compact connected $(n-2)$-dimensional submanifold of $V_{\delta}$. Denote by $l_{c}$ the embedding $M_{c} \hookrightarrow V_{\delta}$ and by $\sigma$ the canonic symplectic 1 -form $\sigma=\xi d x$ in $T^{*} \partial \Omega$, $\omega=-d \sigma$.

Lemma 4.4. There exists $\beta \in \Lambda^{1}(U)$ such that $\beta=0$ in a neighbourhood of $S$,

$$
\|\beta\|_{\rho} \leq C\left|r^{\prime}(\rho)\right|^{N+1 / 2}
$$

where $\|\cdot\|_{\rho}$ is the norm in $T_{\rho}^{*}\left(T^{*} \partial \Omega\right)$ and for $\sigma_{1}=\sigma+\beta$ we have

$$
d\left(l_{c}^{*}\left(F^{*}\right)^{-1} \sigma_{1}\right)=0 \text { for any } c \in(0, \delta)^{n-2} \times(-\delta, \delta) .
$$

Moreover,

$$
\left\{r_{j}, r_{k}\right\}_{1}=0 \text { in } U
$$

where $\{,\}_{1}$ is Poisson's bracket induced by $\omega_{1}=-d \sigma_{1}$. 
Proof. We denote

$$
Z_{j}^{0}=\frac{\partial}{\partial \varphi_{j}}+\sum_{k=1}^{n-1} d_{j k}(\rho) \frac{\partial}{\partial \varphi_{k}}, \quad L_{j}^{0}=\sum_{k=1}^{n-1} c_{j k}(\rho) \frac{\partial}{\partial r_{k}}, \quad j \leq n-1,
$$

and $Z_{j}=\pi_{*}^{-1} Z_{j}^{0}, L_{j}=\pi_{*}^{-1} L_{j}^{0}$. Then the vectors $Z_{j}$ are tangential to $M_{c}$ and $\left|L_{j}\right|_{\rho} \leq C\left|c^{\prime}\right|^{N+1 / 2}$ for any $\rho \in M_{c}$. Here $|\cdot|_{\rho}$ is the norm in $T_{\rho}\left(T^{*} \partial \Omega\right)$. Thus we have

$$
\begin{aligned}
& -d\left(l_{c}^{*}\left(F^{*}\right)^{-1} \sigma\right)\left(Z_{j}, Z_{k}\right)=l_{c}^{*}\left(F^{*}\right)^{-1} \omega\left(Z_{j}, Z_{k}\right) \\
& =\left.\omega\left(X_{r_{j}}, X_{r_{k}}\right)\right|_{r=c}+O\left(\left|c^{\prime}\right|^{N+1}\right)=O\left(\left|c^{\prime}\right|^{N+1}\right) .
\end{aligned}
$$

We write $\left(F^{*}\right)^{-1} \sigma=p d q+t d \zeta+d Q+\tilde{\beta}$ where $\tilde{\beta}$ is a 1 -form with $C^{\infty}$ coefficients. Moreover, from (4.18) we have

$$
d\left(l_{c}^{*} \tilde{\beta}\right)\left(Z_{j}, Z_{k}\right)=d\left(l_{c}^{*}\left(F^{*}\right)^{-1} \sigma\right)\left(Z_{j}, Z_{k}\right)+O\left(\left|c^{\prime}\right|^{N+1}\right)=O\left(\left|c^{\prime}\right|^{N+1}\right) .
$$

On the other hand,

$$
\left(F^{*}\right)^{-1}\left(l_{c}^{*} \sigma\right)=l_{c}^{*}(p d q+d Q+\tilde{\beta})
$$

where

$$
\tilde{\beta}=\sum_{j=1}^{n-2}\left(a_{j} d p_{j}+b_{j} d q_{j}\right)+a_{0} d \zeta+b_{0} d t
$$

and $a_{j}, b_{j}$ are $C^{\infty}$ functions. We expand $a_{j}, b_{j}, j=1, \ldots, n-2$, in Taylor's series up to degree $2 N$ with respect to $p$ and $q$ around the point $p=q=0$ and $b_{0}$ up to degree $2 N+1$. We obtain $\tilde{\beta}=\beta_{1}+\beta_{2}$ where $\beta_{1}=O\left(\left|r^{\prime}\right|^{N+1}\right)$ is the remainder in Taylor's formula. Then from (4.19) we have

$$
d\left(l_{c}^{*} \beta_{2}\right)\left(Z_{j}, Z_{k}\right)=O\left(\left|c^{\prime}\right|^{N+1}\right),
$$

$$
d\left(l_{c}^{*} \beta_{2}\right)=\sum_{j=1}^{n-1} \sum_{k=1}^{n-1} P_{j k}\left(\varphi^{\prime}, t, \sqrt{c_{1}}, \ldots, \sqrt{c_{n-2}}, c_{n-1}\right) d \varphi_{j} \wedge d \varphi_{k}
$$

where $P_{j k}$ are polynomials of $\sqrt{c_{1}}, \ldots, \sqrt{c_{n-2}}$ of degree at most $2 N+1$. In view of (4.20), the definition of $Z_{j}^{0}$ and (4.14) we obtain successively that all coefficients of the polynomials $P_{j k}$ are equal to zero. Hence

$$
d\left(l_{c}^{*} \beta_{2}\right)=0, \quad c \in(0, \delta)^{n-2} \times(-\delta, \delta) .
$$

We denote $\beta=-F^{*} \beta_{1}$. Then $\omega_{1}=-d \sigma_{1}, \sigma_{1}=\sigma+\beta$, is closed on $M_{c}$ for any $c$.

Denote by $X_{f}^{1}$ the Hamilton vector field of the function $f$ with respect to the symplectic form $\omega_{1}$. Let us fix $c \in(0, \delta)^{n-2} \times(-\delta, \delta), p \in M_{c}$. Then

$$
\omega_{1}\left(X_{r_{j}}^{1}, L\right)=\left\langle d r_{j}, L\right\rangle=0, \quad \forall L \in T_{\rho} M_{c} .
$$


Since $T_{\rho} M_{c}$ is Lagrangian with respect to $\omega_{1}$, we obtain $X_{r_{j}}(\rho) \in T_{\rho} M_{c}$. Hence

$$
\left\{r_{j}, r_{k}\right\}_{1}(\rho)=\omega_{1}\left(X_{r_{j}}^{1}, X_{r_{k}}^{1}\right)=0
$$

which proves the lemma.

Let $\rho \in U$. As in [2], consider the map

$$
\mathbb{R}^{n-1} \ni t=\left(t_{1}, \ldots, t_{n-1}\right) \rightarrow T^{t}(\rho)=F_{1}^{t_{1}} \circ \cdots \circ F_{n-1}^{t_{n-1}}(\rho) \in U
$$

where $F_{j}^{t_{j}}$ is the flow of the Hamilton vector field $X_{r_{j}}^{1}$. Let us note that $F_{j}^{t_{j}}$ commute with one another in view of (4.17). Since $M_{c}$ is compact, the stationary group of $t \rightarrow F^{t}(\rho)$ has $n-1$ generators $e_{1}, \ldots, e_{n-1}$ which can be found explicitly in our case. In a neighbourhood $U_{0}$ of $S$ we have $\omega_{1}=\omega$, hence $X_{r_{j}}^{1}=\partial / \partial \varphi_{j}$ where $(\varphi, r)=\pi\left(p, q, \varphi_{n-1}, r_{n-1}\right)$ are the respective polar coordinates. Then in $U_{0}$ we have for $j \leq n-2$

$$
F_{j}^{t_{j}}(\varphi, r)=\left(\varphi_{1}, \ldots, \varphi_{j-1}, \varphi_{j}+t_{j}, \varphi_{j+1}, \ldots, \varphi_{n-1}, r\right) .
$$

In particular, $F_{j}^{t_{j}}: S \rightarrow S$ for $j \leq n-2$ and

$$
e_{j}=(\underbrace{0, \ldots, 0}_{j-1 \text { times }}, 2 \pi, \underbrace{0, \ldots, 0}_{n-j-1 \text { times }}), \quad j \leq n-2,
$$

is a stationary point for $F^{t}(\rho)$. Let $t_{n-1}(r)$ be the smallest positive time such that $F_{n-1}^{t_{n-1}^{(r)}}\left(\rho^{0}\right) \in S$ for $\rho^{0}=(0, r) \in S, r=\left(r_{1}, \ldots r_{n-1}\right)$. Then

$$
\begin{aligned}
F_{n-1}^{t_{n-1}(r)}\left(\rho^{0}\right) & =\left(R_{1}(r), \ldots, R_{n-2}(r), 0, r\right) \\
& =F_{1}^{R_{1}(r)} \circ \cdots \circ F_{n-2}^{R_{n-2}(r)}\left(\rho^{0}\right) .
\end{aligned}
$$

Therefore $e_{n-1}(r)=R(r)=\left(R_{1}(r), \ldots, R_{n-1}(r)\right), R_{n-1}(r)=t_{n-1}(r)$, is a stationary point of $F^{t}$, i.e. $F^{e_{n-1}(r)}(\rho)=\rho$ for any $\rho \in M_{r}$.

Moreover, $R_{j}(r)$ are smooth functions of $\left(p, q, \varphi_{n-1}, r_{n-1}\right)$ and since they do not depend on $\varphi_{j}$, we obtain that $R_{j}$ are smooth functions of $r$ in $[0, \delta]^{n-2} \times[-\delta, \delta]$.

Now, for $(t, r) \in \mathbb{T}^{n-1} \times[0, \delta]^{n-2} \times[-\delta, \delta]$ we set

$$
G_{1}(t, r)=F_{n-1}^{t_{n-1} R_{n-1}(r)}\left(t_{1}+t_{n-1} R_{1}(r), \ldots, t_{n-2}+t_{n-1} R_{n-2}(r), 0, r\right) .
$$

Thus we obtain a smooth map $\pi^{-1} G_{1} \pi$ in the coordinates $\left(p, q, t_{n-1}, r_{n-1}\right)$ :

$$
\pi^{-1} G_{1} \pi\left(p, q, t_{n-1}, r_{n-1}\right)=F_{n-1}^{t_{n-1} R_{n-1}(r)}\left(M\left(\begin{array}{l}
p \\
q
\end{array}\right), 0, r_{n-1}\right)
$$

where $\pi$ is the respective polar change of the coordinates, $M$ is the blockdiagonal matrix $M=\operatorname{diag}\left(M_{1}, \ldots, M_{n-2}\right)$,

$$
M_{j}=\left(\begin{array}{cc}
\cos \left(t_{n-1} R_{j}\right) & -\sin \left(t_{n-1} R_{j}\right) \\
\sin \left(t_{n-1} R_{j}\right) & \cos \left(t_{n-1} R_{j}\right)
\end{array}\right), \quad j=1, \ldots, n-2 .
$$


Since the vectors $e_{j} \in \mathbb{R}^{n-1}, j=1, \ldots, n-1$, are generators of the stationary group of $\mathbb{R}^{n-1} \ni t \rightarrow F^{t}(\rho), \rho \in S$, we obtain that $G_{1}$ is a diffeomorphism of $V_{\delta}$ onto a neighbourhood $U_{\delta}$ of $\widetilde{\mathscr{O}}$ in $T^{*} \partial \Omega$ for $\delta$ small enough. As in [3], we can construct some polar symplectic coordinates $(\varphi, I)$ with respect to $\omega_{1}$ in a neighbourhood of $\{r=0\}$ starting from $(t, r)$ which will give us the smooth exact symplectic transformation $G: V_{\delta} \rightarrow U_{\delta}$ in Proposition 4.2. Unlike [3], we work in a neighbourhood of $\{r=0\}$ and not near a fixed torus and are interested in the smoothness of the respective maps near $\{r=0\}$.

First we shall show that besides the equalities $\left\{r_{j}, r_{k}\right\}_{1}=0, j, k \leq n-1$, we have

$$
\begin{aligned}
& \left\{r_{j}, t_{k}\right\}_{1}=\delta_{j k}, \quad j \leq n-2, k \leq n-1, \\
& \left\{r_{n-1}, t_{j}\right\}_{1}=-R_{j}(r) / R_{n-1}(r), \quad j \leq n-2, \\
& \left\{r_{n-1}, t_{n-1}\right\}_{1}=1 / R_{n-1}(r)
\end{aligned}
$$

and

$$
\left\{t_{j}, t_{k}\right\}_{1}=0 \text { for } j, k \leq n-1 .
$$

Equalities (4.21) follow from

$$
\begin{gathered}
F_{j}^{s} G_{1}(t, r)=G_{1}\left(t+s e_{j}, r\right), \quad j \leq n-2, \\
F_{n-1}^{s} G_{1}(t, r)=G_{1}\left(t_{1}-s R_{1} / R_{n-1}, \ldots, t_{n-2}-s R_{n-2} / R_{n-1}, t_{n-1}+s / R_{n-1}, r\right) .
\end{gathered}
$$

We shall prove equalities (4.22). From Jacobi's identity we have

$$
\begin{gathered}
X_{r_{i}}^{1}\left\{t_{j}, t_{k}\right\}_{1}=0, \quad j, k \leq n-1, i \leq n-2, \\
X_{r_{n-1}}^{1}\left\{t_{j}, t_{k}\right\}_{1}=c_{j k}(r)
\end{gathered}
$$

where $c_{j k}$ are smooth functions of $r \in[0, \delta)^{n-2} \times(-\delta, \delta)$. Hence

$$
\left\{t_{j}, t_{k}\right\}_{1}\left(F_{n-1}^{R_{n-1}(r)}(\rho)\right)=R_{n-1}(r) c_{j k}(r)+\left\{t_{j}, t_{k}\right\}_{1}(\rho), \quad \rho \in S .
$$

On the other hand,

$$
\begin{aligned}
\left\{t_{j}, t_{k}\right\}_{1}(\rho) & =\left\{t_{j}, t_{k}\right\}_{1}\left(F^{e_{n-1}}(\rho)\right)=\left\{t_{j}, t_{k}\right\}_{1}\left(F_{n-1}^{R_{n-1}(r)}(\rho)\right) \\
& =R_{n-1}(r) c_{j k}(r)+\left\{t_{j}, t_{k}\right\}_{1}(\rho), \quad \rho \in S .
\end{aligned}
$$

Thus $c_{j k}(r)=0, j, k \leq n-1$. Moreover, in $U_{0}$

$$
\begin{aligned}
\left\{t_{j}, t_{n-1}\right\}(\rho) & =\left\{\varphi_{j}-\varphi_{n-1} \frac{R_{j}}{R_{n-1}}, \frac{\varphi_{n-1}}{R_{n-1}}\right\} \\
& =\varphi_{n-1}\left(\frac{\partial R_{j}}{\partial r_{n-1}}-\frac{\partial R_{n-1}}{\partial r_{j}}\right) \frac{1}{R_{n-1}^{2}}
\end{aligned}
$$

and since $X_{r_{n-1}}^{1}=\partial / \partial \varphi_{n-1}$ in $U_{0}$, we have $\left\{t_{j}, t_{n-1}\right\}(\rho)=0$ for $\rho \in U_{0}$ and therefore for any $\rho \in U$. Analogously we obtain $\left\{t_{j}, t_{k}\right\}(\rho)=0, \rho \in U, j$, $k \leq n-2$, which proves (4.22). 
We put $G_{2}(t, r)=(\varphi, I)$ where $\varphi_{j}=t_{j}, j \leq n-1, I_{j}=r_{j}, j \leq n-2$, $r_{n-1}=I_{n-1}+Q(I), Q\left(0, t_{0}\right)=-t_{0}$. For the function $Q(I)$ we obtain the equations

$$
\begin{aligned}
&\left\{I_{n-1}, \varphi_{j}\right\}_{1}=-\frac{R_{j}(r)}{R_{n-1}(r)}-\left\{Q(I), t_{j}\right\}_{1} \\
&=-\frac{R_{j}(r)}{R_{n-1}(r)}-\sum_{k=1}^{n-1} \frac{\partial Q}{\partial I_{k}}\left\{I_{k}, t_{j}\right\} \\
&=-\frac{R_{j}(r)}{R_{n-1}(r)}-\frac{\partial Q}{\partial I_{j}}-\frac{\partial Q}{\partial I_{n-1}}\left\{I_{n-1}, \varphi_{j}\right\}_{1}, \quad j \leq n-2, \\
&\left\{I_{n-1}, \varphi_{n-1}\right\}_{1}=\frac{1}{R_{n-1}(r)}-\frac{\partial Q}{\partial I_{n-1}}\left\{I_{n-1}, \varphi_{n-1}\right\}_{1} .
\end{aligned}
$$

That is why we put $\partial Q(I) / \partial I_{j}=P_{j}(I), j=1, \ldots, n-1$, where $P_{j}(I)=$ $-R_{j}(r) / R_{n-1}(r)$ for $j \leq n-2$ and $P_{n-1}(I)=1 / R_{n-1}(r)-1, r=\left(I^{\prime}, I_{n-1}+\right.$ $Q(I))$.

These equations are solvable in a neighbourhood of $\{r=0\}$ since $P_{j}$ are smooth functions and $\partial P_{j} / \partial I_{k}=\partial P_{k} / \partial I_{j}, j, k=1, \ldots, n-1$ (cf. [3]). Indeed, we have

$$
\frac{\partial P_{j}}{\partial I_{k}}=-\left\{t_{k},\left\{r_{n-1}, t_{j}\right\}_{1}\right\}_{1}=-\left\{t_{j},\left\{r_{n-1}, t_{k}\right\}_{1}\right\}_{1}=\frac{\partial P_{k}}{\partial I_{j}} .
$$

Then the map $\pi^{-1} G_{2} G_{1} \pi$ is a diffeomorphism in $V_{\delta}$.

Lemma 4.5. There exists a diffeomorphism $G_{3}: U_{\delta} \rightarrow U$ such that $G_{3}^{*} \omega_{1}=\omega$ and $G_{3}=\mathrm{Id}+G_{4}$ where $\left|G_{4}(\rho)\right| \leq C\left|r^{\prime}(\rho)\right|^{N+1 / 2}$.

Proof. Following Moser [16], consider $\sigma_{t}=\sigma+t \beta, t \in[0,1]$ and $\omega_{t}=$ $\omega-t d \beta$. From (4.15) it follows that $\omega_{t}$ is a nondegenerate 2 -form in $U$ if $U$ is small enough. Let $Y_{t}$ be a vector field determined by $\left.\omega_{t}\right\lrcorner Y_{t}=\beta$ and $\Phi_{t}(\rho)=\exp \left(t Y_{t}\right)(\rho)$. Here $\lrcorner$ denotes the inner product. Then from (4.15) we obtain

$$
\left|\Phi_{t}(\rho)-\rho\right| \leq C\left|r^{\prime}(\rho)\right|^{N+1 / 2}, \quad 0 \leq t \leq 1 .
$$

Moreover, we have

$$
\left.\frac{d}{d t}\left(\Phi_{t}\right)^{*} \omega_{t}=\left(\Phi_{t}\right)^{*}\left(-d \beta+d\left(\omega_{t}\right\lrcorner Y_{t}\right)\right)=0,
$$

hence $G_{3}=\Phi_{1}$ is the diffeomorphism we seek.

Denote $G=G_{3} \pi^{-1} G_{2} G_{1} \pi$. Then $G\left(V_{\delta}\right)=U$ is a neighbourhood of $\tilde{\mathscr{O}}$ in $T^{*} \partial \Omega$ and

$$
G^{*} \omega=\sum_{j=1}^{n-2} d p_{j} \wedge d q_{j}+d I_{n-1} \wedge d \varphi_{n-1} .
$$

It remains to prove (4.6). Denote $\rho_{j}=F_{j}^{R_{j}}\left(\rho_{j-1}\right), 1 \leq j \leq n$, where $\rho_{0} \in S$ has polar coordinates $(0, r(I)), r(I)=\left(r^{\prime}, t_{0} \zeta\right)=\left(I^{\prime}, t_{0} \zeta(I)\right)$. Then $\rho_{n}=\rho_{0}$ 
and $\gamma=\bigcup_{j=1}^{n-1} \gamma_{j}$, where $\gamma_{j}=\left\{F_{j}^{t}\left(\rho_{j-1}\right), 0 \leq t \leq R_{j-1}(r)\right\}$, is a cycle on $M_{r}$. Since the form $\sigma-i d \varphi$ is closed in $U_{\delta}$, we have

$$
\int_{\gamma} \sigma-\int_{\gamma} I d \varphi=\int_{\gamma} \sigma-2 \pi I_{n-1}=c=\text { const . }
$$

Therefore, for $(\varphi, I) \in \Pi^{n-1}$

$$
2 \pi I_{n-1}=\int_{\gamma} \sigma-C=\int_{\gamma} \sigma_{1}+\int_{\gamma} \beta-C=\int_{\gamma} \sigma_{1}+O\left(\left|I^{\prime}\right|^{N+1 / 2}\right)-C .
$$

On the other hand, $\rho_{j}=\left(R_{1}(r), \ldots, R_{j}(r), 0, \ldots, 0, r\right)$ and

$$
\gamma_{j}=\left\{\left(R_{1}(r), \ldots, R_{j-1}(r), t R_{j}(r), 0, \ldots, 0, r\right) ; 0 \leq t \leq 1\right\}
$$

for $j=1, \ldots, n-1$. Denote

$$
\begin{aligned}
L_{j}=\{ & \left(R_{1}\left(s I^{\prime}, t_{0}, \zeta\right), \ldots, R_{j-1}\left(s I^{\prime}, t_{0} \zeta\right),\right. \\
& \left.\left.t R_{j}\left(s I^{\prime}, t_{0} \zeta\right), 0, \ldots, 0, s I^{\prime}, t_{0} \zeta\right) ; 0 \leq s \leq 1,0 \leq t \leq 1\right\}, \quad j \leq n-2, \\
L_{n-1}=\left\{F_{n-1}^{t}\left(R_{1}\left(s I^{\prime}, t_{0} \zeta\right), \ldots, R_{n-1}\left(s I^{\prime}, t_{0} \zeta\right), s I^{\prime}, t_{0} \zeta\right) ;\right. & \left.0 \leq s \leq 1,0 \leq t \leq R_{n-1}(r)\right\}
\end{aligned}
$$

and

$$
\gamma_{\zeta}=\left\{F_{n-1}^{t}(\underbrace{0, \ldots, 0}_{2 n-3 \text { times }}, t_{0} \zeta) ; t \in \mathbb{R}^{1}\right\} .
$$

Note that $\gamma_{\zeta}$ belongs to the cylinder of orbits of $X_{t_{0} \zeta}$. Now we have

$$
\int_{\gamma} \sigma_{1}=\int_{\gamma_{\zeta}} \sigma_{1}-\sum_{j=1}^{n-1} \int_{L_{j}} \omega_{1} .
$$

Moreover, $L_{n-1}$ is isotropic so that $\int_{L_{n-1}} \omega_{1}=0$ while

$$
\begin{aligned}
\sum_{j=1}^{n-2} \int_{L_{j}} \omega_{1} & =\sum_{j=1}^{n-2} \sum_{k=1}^{n-2} \int_{L_{j}} d I_{k} \wedge d \varphi_{k}=\sum_{j=1}^{n-2} \int_{0}^{1} I_{j} R_{j}\left(s I^{\prime}, t_{0} \zeta\right) d s \\
& =\sum_{j=1}^{n-2} \int_{0}^{1} I_{j} \frac{\partial A}{\partial I_{j}}\left(s I^{\prime}, \zeta\right) d s+O\left(\left|I^{\prime}\right|^{N+1 / 2}\right) \\
& =A\left(I^{\prime}, \zeta\right)+O\left(\left|I^{\prime}\right|^{N+1 / 2}\right)
\end{aligned}
$$

since $A(0, \zeta)=0$. Finally, on the cylinder of orbits $L=\left\{F_{n-1}^{t}(0, \ldots, 0, h)\right.$; $0 \leq h \leq \zeta\}$ we have

$$
\begin{aligned}
\int_{\gamma_{\zeta}} \sigma_{1} & =\int_{\gamma_{0}} \sigma_{1}+\int_{L} \omega_{1}=2 \pi t_{0}+\int_{L} d h \wedge d t \\
& =2 \pi t_{0}-\int_{0}^{\zeta} \int_{0}^{R_{n-1}(0 . \zeta)} d t \wedge d h=2 \pi t_{0}-\int_{0}^{\zeta} l(h) d h .
\end{aligned}
$$


Note that for $I^{\prime}=0, I_{n-1}=t_{0}(4.24)$ yields $C=0$ in (4.23), thus $G$ is exact symplectic and

$$
2 \pi I_{n-1}=2 \pi t_{0}-\int_{0}^{\zeta} l(h) d h-A\left(I^{\prime}, \zeta\right)+O\left(\left|I^{\prime}\right|^{N+1 / 2}\right) .
$$

Now if $\zeta_{0}(I)$ is a solution of (4.4)

$$
2 \pi I_{n-1}=2 \pi t_{0}-\int_{0}^{\zeta} l^{0}(h) d h-A^{0}\left(I^{\prime}, \zeta\right), \zeta\left(0, t_{0}\right)=0,
$$

where $l^{0}(\zeta)$ and $A^{0}\left(I^{\prime}, \zeta\right)$ are Taylor's expansions of $l(\zeta)$ and $A\left(I^{\prime}, \zeta\right)$ with respect to $\zeta$ up to degrees $M$ and $M+1$ respectively, $M$ large enough, we obtain

$$
\left|\zeta(\varphi, I)-\zeta_{0}(I)\right| \leq C\left(\left|I^{\prime}\right|^{N+1 / 2}+\left|\zeta_{0}\right|^{M+2}\right)
$$

which proves (4.6).

From the representation of the billiard ball map by means of the interpolating Hamiltonian $\zeta$ and from (4.6) we obtain

$$
B(\varphi, I)=\exp \left(-\sqrt{\zeta} X_{\zeta_{0}}\right)((\varphi, I)+R(\varphi, I)) \text { in } V_{\delta} \cap\{\zeta \geq 0\}
$$

where

$$
R(\varphi, I)=\left(O\left(\left|I^{\prime}\right|^{N-1 / 2}\right)+O\left(\zeta_{0}^{M+2}\right), O\left(\left|I^{\prime}\right|^{N+1 / 2}\right)+O\left(\zeta_{0}^{M+2}\right)\right)
$$

and $R(\varphi, I)=R_{1}\left(\varphi, \sqrt{I^{\prime}}, \sqrt{\zeta}\right)$ where $R_{1}$ is a smooth function of its arguments for $(\varphi, I) \in \mathbb{T}^{n-1} \times \mathbb{D}^{n-1}$. Here we have denoted

$$
\begin{array}{r}
\mathbb{D}^{n-1}=\left\{I \in \mathbb{R}^{n-1} ; 0 \leq I_{j}<\delta, j=1, \ldots, n-2,\left|I_{n-1}-t_{0}\right|<\delta,\right. \\
\left.\zeta_{0}(I)>0,\left|I^{\prime}\right| \leq C \zeta_{0}(I)\right\},
\end{array}
$$

where $C>1$ is such that $\left|\zeta(\varphi, I)-\zeta_{0}(I)\right| \leq \frac{1}{2}\left(\zeta_{0}(I)\right)^{M+2}$ for $I \in \mathbb{D}^{n-1}$ and $\varphi \in \mathbb{T}^{n-1}$ (here we have used (4.5)).

Then we have

$$
B(\varphi, I)=\exp \left(-\sqrt{\zeta_{0}} X_{\zeta_{0}}\right)((\varphi, I)+g(\varphi, I))
$$

for $(\varphi, I) \in \mathbb{T}^{n-1} \times \mathbb{D}^{n-1}$ where

$$
g(\varphi, I)=\left(O\left(\left|I^{\prime}\right|^{N-1 / 2}\right), O\left(\left|I^{\prime}\right|^{N+1 / 2}\right)\right)+O\left(\zeta_{0}^{M+2}\right)
$$

and $g$ is symplectic in $\mathbb{T}^{n-1} \times \mathbb{D}^{n-1}$.

4.3. Denote

$$
\begin{array}{r}
\mathbb{D}_{a}^{n-1}=\left\{I \in \mathbb{R}^{n-1}, C_{1} a \leq I_{j} \leq C_{2} a, j=1, \ldots, n-2,\right. \\
\left.C_{3} a^{2 b} \leq \zeta_{0}(I) \leq C_{4} a^{2 b}\right\}
\end{array}
$$

where $0<C_{1}<1<C_{2}, 0<C_{3}<1<C_{4}, 0<b<1 / 2$ small enough. Henceforth $C_{j}$ will be constants which do not depend on $a$ but they will 
depend on the concrete circumstances. Note that for $a \leq a_{0}, a_{0}$ small enough, we have $\mathbb{D}_{a}^{n-1} \subset \mathbb{D}^{n-1}$. We shall denote $A_{a}^{n-1}=\mathbb{T}^{n-1} \times \mathbb{D}_{a}^{n-1}$.

Denote for $\Omega \subset \mathbb{R}^{m}, \rho>0$,

$$
\Omega+\rho=\left\{z \in \mathbb{C}^{m} ; \operatorname{dist}(z, \Omega) \leq \rho\right\}
$$

and set $H^{0}(I)=-\frac{2}{3}\left(\zeta_{0}(I)\right)^{3 / 2}, H(\varphi, I)=-\frac{2}{3}(\zeta(\varphi, I))^{3 / 2}$.

Proposition 4.6. For $a_{0}$ small enough we have

$$
\begin{gathered}
\left\|H_{I I}^{0}(I)\right\|,\left\|\left(H_{I I}^{0}(I)\right)^{-1}\right\| \leq C_{1}^{\prime} a^{-b}, \quad I \in \mathbb{D}_{a}^{n-1}+\rho, \\
\left|\operatorname{det} H_{I I}^{0}(I)\right| \geq C_{2}^{\prime} a^{b(n-3)}
\end{gathered}
$$

for each $a \in\left(0, a_{0}\right), 0<\rho \leq a / 2$, where $\|\cdot\|$ is the norm of the respective $(n-1) \times(n-1)$-matrix. Moreover, the map

$$
\mathbb{D}_{a}^{n-1}+\rho \ni I \rightarrow H_{I}^{0}(I)
$$

is a diffeomorphism.

Proof. We have $H_{I}^{0}(I)=-\zeta_{0}^{1 / 2} \operatorname{grad} \zeta_{0}(I)$. But

$$
\operatorname{grad} \zeta_{0}(I)=-\left(l^{0}\left(\zeta_{0}\right)+A_{\zeta}^{0}\left(I^{\prime}, \zeta_{0}\right)\right)^{-1}\left(A_{I^{\prime}}^{0}\left(I^{\prime}, \zeta_{0}\right),-1\right),
$$

thus

$$
H_{I}^{0}=\zeta_{0}^{1 / 2}\left(l^{0}\left(\zeta_{0}\right)+A_{\zeta}^{0}\left(I^{\prime}, \zeta_{0}\right)\right)^{-1}\left(A_{I^{\prime}}^{0}\left(I^{\prime}, \zeta_{0}\right),-1\right) .
$$

Set $K=\zeta_{0}\left(l^{0}\left(\zeta_{0}\right)+A_{\zeta}^{0}\left(I^{\prime}, \zeta_{0}\right)\right)^{-2}$. We have $\partial K(0) / \partial \zeta_{0} \neq 0$ and this allows us to regard the map $I \rightarrow H_{I}^{0}(I)$ as a composition of the following three maps:

$$
\left(I^{\prime}, I_{n-1}\right) \rightarrow\left(I^{\prime}, \zeta_{0}\right) \rightarrow\left(I^{\prime}, K\right) \rightarrow H_{I}^{0}(I) .
$$

This gives us the representation

$$
H_{I I}^{0}(I)=\frac{\partial H_{I}^{0}}{\partial\left(I^{\prime}, K\right)} \frac{\partial\left(I^{\prime}, K\right)}{\partial\left(I^{\prime}, \zeta_{0}\right)} \frac{\partial\left(I^{\prime}, \zeta_{0}\right)}{\partial\left(I^{\prime}, I_{n-1}\right)} .
$$

The first two maps are diffeomorphisms in a sufficiently small neighbourhood of $\mathscr{O}$ and for the respective functional matrices we have

$$
\begin{gathered}
\frac{\partial\left(I^{\prime}, K\right)}{\partial\left(I^{\prime}, \zeta_{0}\right)}=\left(\begin{array}{cc}
E_{n-2} & O_{n-2} \\
K_{I^{\prime}} & \partial K / \partial \zeta_{0}
\end{array}\right) \\
\frac{\partial\left(I^{\prime}, \zeta_{0}\right)}{\partial\left(I^{\prime}, I_{n-1}\right)}=\left(\begin{array}{cc}
E_{n-2} & O_{n-2} \\
\operatorname{grad}_{I^{\prime}} \zeta_{0} & \partial \zeta_{0} / \partial I_{n-1}
\end{array}\right)
\end{gathered}
$$

where $E_{n-2}$ is the unit $(n-2)$-matrix and $O_{n-2}$ is the zero $(n-2)$-dimensional column matrix. Thus it remains to consider the matrix $\partial H_{I}^{0} / \partial\left(I^{\prime}, K\right)$ for $I \in$ $\mathbb{D}_{a}^{n-1}+\rho$, i.e. when the variables $\left(I^{\prime}, K\right)$ run over a set of the form

$$
\left[\widetilde{C}_{1} a, \widetilde{C}_{2} a\right]^{n-2} \times\left[\widetilde{C}_{3} a^{2 b}, \widetilde{C}_{4} a^{2 b}\right]+\widetilde{C}_{5} \rho .
$$


We have $H_{I}^{0}=K^{1 / 2}\left(A_{I^{\prime}}^{0}\left(I^{\prime}, \zeta_{0}\right),-1\right)$ where $\zeta_{0}=K A^{1}\left(I^{\prime}, K\right)$ and the function $A^{1}\left(I^{\prime}, K\right)$ satisfies $A^{1}(0,0) \neq 0$. Then for the entries of the matrix $\partial H_{I}^{0} / \partial\left(I^{\prime}, K\right)$ we have

$$
A_{j k}=K^{1 / 2}\left(\frac{\partial^{2} A^{0}\left(I^{\prime}, \zeta_{0}\right)}{\partial I_{j} \partial I_{k}}+K \frac{\partial A^{0}\left(I^{\prime}, \zeta_{0}\right)}{\partial I_{j}} \cdot \frac{\partial A^{1}\left(I^{\prime}, K\right)}{\partial I_{k}}\right)
$$

for $1 \leq j, k \leq n-2$,

$$
\begin{aligned}
& A_{j, n-1}=\frac{1}{2} K^{-1 / 2} \frac{\partial A^{0}\left(I^{\prime}, \zeta_{0}\right)}{\partial I_{j}}+K^{1 / 2} \frac{\partial^{2} A^{0}\left(I^{\prime}, \zeta_{0}\right)}{\partial I_{j} \partial \zeta}\left(A^{1}\left(I^{\prime}, K\right)+K \frac{\partial A^{1}\left(I^{\prime}, K\right)}{\partial K}\right) \\
& \quad \text { for } j=1, \ldots, n-2, \\
& A_{n-1, k}=0 \text { for } k=1, \ldots, n-2, A_{n-1, n-1}=-\frac{1}{2} K^{-1 / 2} .
\end{aligned}
$$

Now it is easy to see that the validity of Proposition 4.6 follows from the invertibility of the matrix $A_{I^{\prime} I^{\prime}}^{0}\left(I^{\prime}, \zeta\right)$ for $I^{\prime}, \zeta$ small enough which is a consequence of the nondegeneracy assumption (2.3). This completes the proof of Proposition 4.6.

\section{MAIN THEOREM}

Denote $\Omega_{a}=\left\{\omega=H_{I}^{0}(I) ; I \in \mathbb{D}_{a}^{n-1}\right\}$ and let the numbers $\sigma$ and $\gamma$ be such that $\sigma>n-1$ and $\gamma>0$.

Then denote

(5.1) $\Omega_{a}^{\gamma}=\left\{\omega \in \Omega_{a} ;\left|\left\langle\omega, k^{\prime}\right\rangle-k_{n}\right| \geq \gamma|k|^{-\sigma}\right.$ for any $\left.k=\left(k^{\prime}, k_{n}\right) \in \mathbb{Z}^{n} \backslash\{0\}\right\}$.

The next lemma shows that the family of invariant tori we are going to obtain has a positive Lebesgue measure.

Lemma 5.1. Let $\gamma<\widetilde{C}_{6} a^{1+l b}, \widetilde{C}_{6}>0, l>0$. Then for a small enough we have

$$
\operatorname{mes} \Omega_{a}^{\gamma} \geq\left(1-\tilde{c}_{1} a^{l b}\right) \tilde{c}_{2} a^{n-2+b} .
$$

Proof. By analytic-geometrical arguments we obtain mes $\Omega_{a} \geq \widetilde{C}_{7} a^{n-2+b}$. Then by arguments close to those in [1] we find $\operatorname{mes}\left(\Omega_{a} \backslash \Omega_{a}^{\gamma}\right) \leq \widetilde{C}_{8} \gamma a^{n-3+b}$ where $\widetilde{C}_{8}$ depends only on $n$ and $\sigma$. This yields the desired inequality.

Denote by $\sigma_{\gamma}(\varphi, I)$ the map $(\varphi, I) \rightarrow(\varphi, \gamma I)$ and by $\|\cdot\|_{s, \top^{n-1} \times \Omega}, s>0$, the respective Hölder norms of the functions in $T^{n-1} \times \Omega$ as well as

$$
\|f\|_{s, \mathbb{T}^{n-1} \times \Omega ; \gamma}=\left\|f \circ \sigma_{y}\right\|_{s, \sigma_{\%}^{-1}\left(\mathbb{T}^{n-1} \times \Omega\right)}
$$

(see [19]).

Let $C_{5} a^{1+l b}<\gamma_{0}<C_{6} a^{1+l b}, 1<C_{5}<C_{6}, l>0$, and denote $\gamma=\gamma_{0} a^{3 b}$.

Theorem 1 follows from the following theorem.

Theorem 5.2. Let $N \geq 2,0<a<a_{0}$ where $a_{0}$ is small enough. Then there exists an exact symplectic diffeomorphism $U: \mathrm{A}_{a}^{n-1} \rightarrow \mathrm{A}_{a}^{n-1}$ and a function $K$, 
$K(I)>0$ in $\mathbb{D}_{a}^{n-1}$, of class $C^{\infty}$ such that

$$
U^{-1} B U(\varphi, I)=\left(\varphi+\tau_{I}(I), I\right), \quad \tau(I)=-\frac{2}{3} K(I)^{3 / 2},
$$

for any $\varphi \in \mathbb{T}^{n-1}, I \in \mathbb{D}_{a, \gamma_{0}}^{n-1}=\left\{I \in \mathbb{D}_{a}^{n-1} ; \tau_{I}(I) \in \Omega_{a}^{\gamma_{0}}\right\}$. Moreover,

$$
\left\|K-\zeta_{0}\right\|_{p, \mathbb{D}_{a}^{n-1} ; \gamma} \leq C_{p} a^{N-1 / 2-(l+2) b}
$$

and the generating function $S(\theta, I)$ of $U$ satisfies

$$
\|S\|_{p, \mathbf{A}_{a}^{n-1} ; z} \leq C_{p} a^{N-1 / 2-(l+1) b} \quad \text { for any } p \geq 0 .
$$

In order to prove Theorem 5.2 we reduce the problem to finding invariant tori for the flow of a suitable Hamiltonian. For this purpose we use some arguments from $[4,5]$.

Now we shall use the fact that the map Id $+g$ from (4.25) is exact symplectic. This is a consequence of (4.25) and the following assertion proved in [6]:

Proposition 5.3. The billiard ball map $B$ is exact symplectic in $V_{\delta}$.

Let $\eta \in C^{\infty}\left(\mathbb{R}^{1}\right), \eta=0$ in a neighbourhood of $0, \eta=1$ in a neighbourhood of $2 \pi$ and let $\varphi_{t}$ be exact symplectic with a generating function $\eta(t) S$ where $S$ generates $\varphi_{2 \pi}=\mathrm{Id}+g$. Denote by $B_{t}$ the exact symplectic map $B_{t}=$ $\exp \left(t X_{H^{0}}\right) \circ \varphi_{t}, t \in \mathbb{R}^{1}$. Let $\xi_{t}=d B_{t} / d t \circ\left(B_{t}\right)^{-1}$ be the respective vector field which is well defined for $t \in \mathbb{T}^{1}$ since $\xi_{t}=X_{H^{0}}$ in some neighbourhoods of $t=0$ and $t=2 \pi$. Following Douady [4], we obtain $\left.\omega_{n-1}\right\lrcorner \xi_{t}=d h_{t}$ for a suitable $C^{\infty}$ function $h_{t}, t \in \mathbb{T}^{1}$. Moreover, using (4.25) we have

$$
\begin{aligned}
(5.5) h_{t}(\varphi, I)= & H^{0}(I)+\sqrt{\zeta_{0}} \sum_{|\alpha|=2 N+1}\left(I^{\prime}\right)^{\alpha / 2} Q_{\alpha}\left(t, \varphi, \sqrt{I_{1}}, \ldots, \sqrt{I_{n-2}}, \sqrt{\zeta_{0}}\right) \\
& +\zeta_{0}^{M+5 / 2} Q^{\prime}\left(t, \varphi, \sqrt{I_{1}}, \ldots, \sqrt{I_{n-2}}, \sqrt{\zeta_{0}}\right)
\end{aligned}
$$

for $(\varphi, I) \in \mathrm{A}_{a}^{n-1}$ where $Q_{c}, Q^{\prime}$ are smooth functions of $\left(t, \varphi, \sqrt{I_{1}}, \ldots\right.$, $\left.\sqrt{I_{n-2}}, \sqrt{\zeta_{0}}\right)$

Denote $y^{\prime}=\varphi, y=\left(y^{\prime}, y_{n}\right), \eta^{\prime}=I, \eta=\left(\eta^{\prime}, \eta_{n}\right)$ where the variable $\eta_{n}$ runs over a neighbourhood of 0 in $\mathbb{R}^{1}$ and $y_{n} \in \mathbb{T}^{1}$. Set

$$
\widetilde{H}^{0}(\eta)=H^{0}\left(\eta^{\prime}\right)+\eta_{n}, \quad \tilde{H}(y, \eta)=h_{y_{n}}\left(y^{\prime}, \eta^{\prime}\right)+\eta_{n} .
$$

Denote by $\left(F^{t}\right)_{t \in \mathbb{R}^{\prime}}$ the flow of $X_{\widetilde{H}}$ and let $A^{\prime}=\left\{(y, \eta) \in \mathrm{A}_{a}^{n} ; \widetilde{H}(y, \eta)=0\right.$, $\left.y_{n}=0\right\}$ where $A_{a}^{n}=\mathbb{T}^{n} \times \mathbb{D}_{a}^{n}$,

$$
\begin{aligned}
\mathbb{D}_{a}^{n}=\left\{\eta \in \mathbb{R}^{n} ; C_{7} a \leq \eta_{j} \leq C_{8} a, j=1\right. & , \ldots, n-2, \\
& \left.C_{9} a^{2 b} \leq \zeta_{0}\left(\eta^{\prime}\right) \leq C_{10} a^{2 b},\left|\eta_{n}\right| \leq C_{11}\right\}
\end{aligned}
$$

and $C_{j}$ are some positive constants such that $C_{7}<1<C_{8}, C_{9}<1<C_{10}$. It is easy to see that $A^{\prime}$ has the form

$$
\mathrm{A}^{\prime}=\left\{\left(y^{\prime}, 0, \eta^{\prime},-H^{0}\left(\eta^{\prime}\right)\right) ;\left(y^{\prime}, \eta^{\prime}\right) \in \mathrm{A}_{a}^{n-1}\right\} .
$$


Denote by $l: \mathrm{A}_{a}^{n-1} \rightarrow \mathrm{A}$ the map

$$
\imath\left(y^{\prime}, \eta^{\prime}\right)=\left(y^{\prime}, 0, \eta^{\prime},-h_{0}\left(y^{\prime}, \eta^{\prime}\right)\right)=\left(y^{\prime}, 0, \eta^{\prime},-H^{0}\left(\eta^{\prime}\right)\right) .
$$

It is easy to see that $F^{2 \pi}: A^{\prime} \rightarrow A^{\prime}$ satisfies

$$
l^{-1} \circ F^{2 \pi} \circ l=B .
$$

Denote $H_{0}^{\prime}=\widetilde{H}^{0}+\left(\widetilde{H}^{0}\right)^{2}, H^{\prime}=\widetilde{H}+\widetilde{H}^{2}$.

Now, from Proposition 4.6 we obtain

$$
\left\|\frac{\partial^{2} H_{0}^{\prime}}{\partial \eta^{2}}\right\|,\left\|\left(\frac{\partial^{2} H_{0}^{\prime}}{\partial \eta^{2}}\right)^{-1}\right\| \leq C a^{-b} \text { in } \mathbb{D}_{a}^{n}+\rho
$$

where $0<a \leq a_{0}, b>0$ small enough are fixed and $\rho \leq a / 2$. Moreover, in $\mathrm{A}_{a}^{n}$ we have

$$
\begin{aligned}
H^{\prime}(y, \eta)-H_{0}^{\prime}(\eta) & =\left(h_{y_{n}}\left(y^{\prime}, \eta^{\prime}\right)-H^{0}\left(\eta^{\prime}\right)\right)\left(1+\widetilde{H}(y, \eta)+H^{0}\left(\eta^{\prime}\right)\right) \\
& =a^{b} O\left(\left|\eta^{\prime \prime}\right|^{N+1 / 2}\right)
\end{aligned}
$$

where $\eta^{\prime \prime}=\left(\eta_{1} \ldots, \eta_{n-2}\right)$ which follows from (5.5) for $M$ large enough.

Introduce the sets $\Omega^{\prime}=\left\{\omega=\operatorname{grad} H_{0}^{\prime}(\eta) ; \eta \in \mathbb{D}_{a}^{n}\right\}$ and $\Omega_{\gamma_{1}}^{\prime}=\left\{\omega \in \Omega^{\prime}\right.$; $|\langle\omega, k\rangle| \geq \gamma_{1}|k|^{-\sigma}$ for any $\left.k \in \mathbb{Z}^{n} \backslash\{0\}\right\}$ where $\sigma$ is as above and $\gamma_{1}=$ $C \gamma a^{-3 b}=C \gamma_{0}, 0<C<1$.

We reduce the proof of Theorem 5.2 to

Theorem 5.4. Let $H_{0}^{\prime}(\eta)$ be an analytic function in $\mathbb{D}_{a}^{n}+\rho$ such that

$$
\left\|\frac{\partial^{2} H_{0}^{\prime}}{\partial \eta^{2}}\right\|_{\mathbb{D}_{a}^{n}+\rho},\left\|\left(\frac{\partial^{2} H_{0}^{\prime}}{\partial \eta^{2}}\right)^{-1}\right\|_{\mathbb{D}_{a}^{n}+\rho} \leq R, \quad R \geq 1,
$$

and let $\partial H_{0}^{\prime} / \partial \eta: \mathbb{D}_{a}^{n}+\rho \rightarrow \mathbb{C}^{n}$ be invertible on $\mathbb{D}_{a}^{n}+\rho$.

Then for any fixed $\lambda>\sigma+1>n, \alpha>1, \alpha \notin \Lambda=\{i / \lambda+j ; i, j \geq 0$ integer $\}$ there exists $\delta \leq C_{1} R^{-3}$ such that if $H^{\prime} \in C^{\infty}\left(\mathrm{A}_{a}^{n}\right)$ and

$$
R\left\|H^{\prime}-H_{0}^{\prime}\right\|_{s, \mathbf{A}_{a}^{n} ; \gamma_{1}} \leq \gamma_{1}^{2} \delta, \quad s=\alpha \lambda+\lambda+\sigma, 0<\gamma_{1} \leq \rho / R,
$$

then

(i) there exists a $C^{\infty}$ function $\widetilde{S}(Y, \eta)$ and a nondegenerate Hamiltonian $K^{\prime}(\eta)$ such that

$$
\left.H^{\prime}\left(Y, \eta-\widetilde{S}_{Y}(Y, \eta)\right)\right|_{\mathrm{A}_{a, \gamma_{1}}^{n}}=K^{\prime}(\eta)
$$

where $\mathrm{A}_{a, \gamma_{1}}^{n}=\mathbb{T}^{n} \times \mathbb{D}_{a, \gamma_{1}}^{n}$ and $\mathbb{D}_{a, \gamma_{1}}^{n}=\left\{\eta \in \mathbb{R}^{n} ; K_{\eta}^{\prime}(\eta) \in \Omega_{\gamma_{1}}^{\prime}\right\}$;

(ii) $\|\widetilde{S}\|_{\tilde{\beta}_{,}, \mathbf{A}_{a}^{n} ; \gamma_{1}} \leq C_{\beta} \gamma_{1}^{-1} R^{\beta+1}\left\|H^{\prime}-H_{0}^{\prime}\right\|_{p, \mathbf{A}_{a}^{n} ; \gamma_{1}}$ where $p=\beta \lambda+\lambda+\sigma, \widetilde{\beta}=$ $\beta-(\lambda-\sigma) / \lambda \notin \Lambda, \beta \geq \alpha$;

(iii) $\tilde{S}$ generates a canonic map $\tilde{T}$ on $A_{a}^{n}$ for $\left|\widetilde{S}_{Y \eta}\right|$ small enough. 
In fact, Theorem 5.4 is proved in [19, Theorem A]. We just follow the dependence of the various constants in [19] on $R$ and $\rho$ as well as the exponent of $\gamma_{1}$ in (ii) (for more details see the Appendix).

Proof of Theorem 5.2. First we estimate $H^{\prime}-H_{0}^{\prime}$. Using (5.9) and the fact that $H^{\prime}-H_{0}^{\prime}$ is a smooth function of $\left(y, \sqrt{\eta_{1}}, \ldots, \sqrt{\eta_{n-2}}, \sqrt{\zeta_{0}}, \eta_{n}\right)$ in $A_{a}^{n}$, we obtain

$$
R\left\|H^{\prime}-H_{0}^{\prime}\right\|_{p, \mathrm{~A}_{a}^{n} ; \gamma_{1}} \leq C a^{N+1 / 2} \leq \gamma_{1}^{2} \delta \text { for any } p>0
$$

where $\delta=a^{4 b}, R=C a^{-b}, N \geq 2$ and $0<b<1 /(4 l+8)$. Thus Theorem 5.4 holds for $H^{\prime}$ and $H_{0}^{\prime}$ and from (ii) we obtain

$$
\|\widetilde{S}\|_{p, \mathrm{~A}_{a}^{n} ; \gamma_{1}} \leq C_{p} a^{N-1 / 2-(p+l+1) b} \text { for any } p>0 .
$$

Note that $\widetilde{S}_{Y \eta}$ is small enough if $a_{0}$ is small enough and $N \geq 2$ since in view of (5.13) we have $\left|\widetilde{S}_{Y \eta}\right| \leq C a^{N-3 / 2-(2+2 l) b}$ in $\mathrm{A}_{a}^{n}$. Then $\widetilde{S}$ generates a canonic map $\widetilde{T}$, i.e.

$$
\text { graph } \widetilde{T}=\left\{\left(Y-\widetilde{S}_{\eta}(Y, \eta), \eta ; Y, \eta-\widetilde{S}_{Y}(Y, \eta)\right)\right\} \text {. }
$$

Now we shall concentrate our efforts on the construction of the function $K$ and the symplectic map $U$ of Theorem 5.2 starting from the nongenerate Hamiltonian $K^{\prime}$ and the symplectic transformation $\widetilde{T}$.

First we shall need some preliminaries.

Let us denote $K_{1}(y, \eta)=\widetilde{H}(\widetilde{T}(y, \eta))$. Then $K_{1}+K_{1}^{2}=K^{\prime}$ and $K_{1}$ does not depend on $y$ for any $\eta \in \mathbb{D}_{a, \gamma}^{n}$. Set $K_{2}(\eta)=K_{1}(0, \eta)$.

The manifold $\Sigma_{0}=\left\{(y, \eta) \in \mathrm{A}_{a}^{n} ; \widetilde{H}(y, \eta)=0\right\}$ is contained in $\left\{(y, \eta) \in \mathrm{A}_{a}^{n}\right.$; $\left.H^{\prime}(y, \eta)=0\right\}$. Moreover, if $\Lambda_{\eta} \cap \Sigma_{0} \neq \varnothing$ for some invariant torus $\Lambda_{\eta}=$ $\widetilde{T}\left(T^{n} \times\{\eta\}\right)$, then $\Lambda_{\eta} \subset \Sigma_{0}$ and

$$
\left.F^{t}\right|_{\Lambda_{\eta}}=\left.\exp \left(t X_{H^{\prime}}\right)\right|_{\Lambda_{\eta}} \text { where } F^{t}(\rho)=\exp \left(t X_{\widetilde{H}}\right)(\rho)=\left(y^{t}(\rho), \eta^{t}(\rho)\right) .
$$

Denote $\widetilde{\Sigma}=\widetilde{T}^{-1}\left(\Sigma_{0}\right)=\left\{(y, \eta) \in \mathrm{A}_{a}^{n} ; K_{1}(y, \eta)=0\right\}$.

Since $\tilde{T}$ is a canonic map, we have

$$
F^{t}(\widetilde{T}(y, \eta))=\widetilde{T}\left(y+t \operatorname{grad} K_{2}(\eta), \eta\right)
$$

for $(y, \eta) \in \mathrm{A}_{a, \gamma_{1}}^{n} \cap \widetilde{\Sigma}$, i.e. when $y \in \mathbb{T}^{n}, \eta \in \mathbb{D}_{a, \gamma_{1}}^{n}$ and $K_{2}(\eta)=0$. We write down $\widetilde{T}(y, \eta)=(p(y, \eta), q(y, \eta))$. From (5.6) we have $\dot{y}_{n}=\partial \widetilde{H} / \partial \eta_{n}=1$, thus $y_{n}^{t}=y_{n}^{0}+t$ and we have

$$
p_{n}(y, \eta)+t=p_{n}\left(y+t \operatorname{grad} K_{2}(\eta), \eta\right) \quad \text { for any } t \in \mathbb{R}^{1} .
$$

As in [4] we conclude that $\operatorname{grad}_{y} p_{n}(y, \eta)$ does not depend on $y \in \mathbb{T}^{n}$ if $\eta \in \mathbb{D}_{a, y_{1}}^{n}$ and $K_{2}(\eta)=0$. Therefore, $p_{n}(y, \eta)=y_{n}+f(\eta)$ for some function $f$ on $\mathbb{D}_{a, \gamma_{1},}^{n}$. On the other hand,

$$
\widetilde{T}\left(-\widetilde{S}_{\eta}(0, \eta), \eta\right)=\left(0, \eta-\widetilde{S}_{Y}(0, \eta)\right), \quad(0, \eta) \in \mathrm{A}_{a, \gamma_{1}}^{n} \cap \widetilde{\Sigma},
$$


thus

$$
-\widetilde{S}_{\eta_{n}}(0, \eta)+f(\eta)=p_{n}\left(-\widetilde{S}_{\eta}(0, \eta), \eta\right)=0
$$

and $f(\eta)=\widetilde{S}_{\eta_{n}}(0, \eta)$. From $(5.15)$ we obtain

$$
y_{n}+f(\eta)+t=y_{n}+t \frac{\partial K_{2}}{\partial \eta_{n}}(\eta)+f(\eta)
$$

thus

$$
\frac{\partial K_{2}}{\partial \eta_{n}}(\eta)=1, \quad \eta \in \mathbb{D}_{a, \gamma_{1}}^{n}
$$

Denote $g(\eta)=\widetilde{S}_{\eta}(0, \eta), \eta \in \mathbb{D}_{a}^{n}$; then the map $T_{1}(y, \eta)=(y-g(\eta), \eta)$ is symplectic with a generating function $\widetilde{S}(0, \eta)$. Moreover, the map $\widetilde{T}$ 。 $T_{1}$ is generated by the function $\widetilde{S}(Y, \eta)-\widetilde{S}(0, \eta)$ which satisfies (ii) and $\tilde{H}\left(\tilde{T}\left(T_{1}(y, \eta)\right)\right)=K_{2}(\eta)$ for $\eta \in \mathbb{D}_{a, \gamma_{1}}^{n}$.

Thus we can suppose that

$$
p_{n}(y, \eta)=y_{n}
$$

for $(y, \eta) \in \mathrm{A}_{a, \gamma_{1}}^{n} \cap \widetilde{\Sigma}$ and $y_{n}$ small enough.

Then Theorem 5.4(i), (5.14) and (5.17) yield $p_{n}\left(y^{\prime}, 0, \eta\right)=0$ and

$$
F^{2 \pi}\left(\widetilde{T}\left(y^{\prime}, 0, \eta\right)\right)=\widetilde{T}\left(y^{\prime}+\operatorname{grad}_{\eta^{\prime}} K_{2}(\eta), 2 \pi, \eta\right)
$$

for $\left(y^{\prime}, 0, \eta\right) \in \widetilde{\Sigma} \cap A_{a, \gamma_{1}}^{n}$.

We turn now to the construction of the function $K(I)$ in Theorem 5.2. First we prove that

$$
\left\|K_{2}-\widetilde{H}^{0}\right\|_{p, \mathbb{D}_{a}^{n} ; \gamma_{2}} \leq C_{p} a^{N-1 / 2-(l+1) b} \text { for any } p \geq 0, \gamma_{2}=\gamma_{1} a^{b} .
$$

Indeed, we have

$$
\begin{aligned}
K_{2}(\eta)-\widetilde{H}^{0}(\eta)= & \left(\widetilde{H}\left(0, \eta-\widetilde{S}_{Y}(0, \eta)\right)-\widetilde{H}^{0}\left(\eta-\widetilde{S}_{Y}(0, \eta)\right)\right) \\
& +\left(\widetilde{H}^{0}\left(\eta-\widetilde{S}_{Y}(0, \eta)\right)-\widetilde{H}^{0}(\eta)\right) .
\end{aligned}
$$

We evaluate the first addend by (5.12) and (5.13) using the following estimate for the norm of a composite function $F \circ G$ :

$$
\|F \circ G\|_{p, \gamma_{1}} \leq\|F\|_{p, \gamma_{1}} \sum_{\alpha=0}^{p-1} c_{p, \alpha}\|D G\|_{\alpha, \gamma_{1}}^{p-\alpha}, \quad p \geq 1
$$

where $D G$ is the matrix of the first derivatives of $G$ and $C_{p, p-1}=1$. For the sake of simplicity we have dropped out the dependence of the above norms on the domain $\mathbb{D}_{a}^{n}$.

For the second term we have

$$
\widetilde{H}^{0}\left(\eta-\widetilde{S}_{Y}(0, \eta)\right)-\widetilde{H}^{0}(\eta)=-\left\langle\widetilde{S}_{Y}(0, \eta), \int_{0}^{1}\left(\operatorname{grad} \widetilde{H}^{0}\right)\left(\eta-t \widetilde{S}_{Y}(0, \eta)\right) d t\right\rangle
$$


whose $\left(p, \gamma_{1}\right)$-norm can be estimated by $C_{p} a^{\beta}, \beta=N-1 / 2-(p+l+1) b$, using again (5.13) and (5.20). This proves (5.19).

Now, for $N \geq 2$, we can use the implicit function theorem to solve the equation $K_{2}(\eta)=0$ with respect to $\eta_{n}$. We find a smooth function $\tau\left(\eta^{\prime}\right)$ choosing the constants $C_{j}$ in the definition of $\mathbb{D}_{a}^{n-1}$ and $\mathbb{D}_{a}^{n}$ appropriately so that the set $\Sigma_{1}=\left\{\eta ; K_{2}(\eta)=0\right\}$ can be represented as $\Sigma_{1}=\left\{\left(\eta^{\prime},-\tau\left(\eta^{\prime}\right)\right)\right.$; $\left.\eta^{\prime} \in \mathbb{D}_{a}^{n-1}\right\}$. Moreover, making use of (5.19) and (5.20), we find

$$
\left\|\tau+\frac{2}{3} \zeta_{0}^{3 / 2}\right\|_{p, \mathbb{D}_{a}^{n-1} ; \gamma_{2}} \leq C_{p} a^{N-1 / 2-(l+1) b}, \quad p \geq 0 .
$$

Thus $\tau<0$ in $\mathbb{D}_{a}^{n-1}$ and the function $K\left(\eta^{\prime}\right)=\left(-\frac{3}{2} \tau\left(\eta^{\prime}\right)\right)^{2 / 3}$ is smooth and positive in $\mathbb{D}_{a}^{n-1}$. We shall prove that $K$ satisfies the requirements of Theorem 5.2. First, in view of $(5.21)$ and the equality

$$
K-\zeta_{0}=\zeta_{0}\left(\left(1-\frac{3}{2} \zeta_{0}^{-3 / 2} R\right)^{2 / 3}-1\right), \quad R=\tau+\frac{2}{3} \zeta_{0}^{3 / 2},
$$

we obtain

$$
\left\|K-\zeta_{0}\right\|_{p, \mathbb{D}_{a}^{n-1} ; \gamma_{2}} \leq C_{p} a^{N-1 / 2-(l+2+2 p) b}
$$

which yields $(5.3)$ for $\gamma=\gamma_{2} a^{2 b}=\gamma_{1} a^{3 b}$.

It remains to construct the symplectic diffeomorphism $U$ of Theorem 5.2. We shall write down explicitly the generating function of $U$ starting from $\widetilde{S}$.

Lemma 5.5. Suppose that $\eta^{\prime} \in \mathbb{D}_{a, \gamma_{0}}^{n-1}=\left\{\eta^{\prime} \in \mathbb{D}_{a}^{n-1} ; \operatorname{grad} \tau\left(\eta^{\prime}\right) \in \Omega_{\gamma_{0}}\right\}$. Then $\left(\eta^{\prime},-\tau\left(\eta^{\prime}\right)\right) \in \mathbb{D}_{a, \gamma_{1}}^{n}$ and $\operatorname{grad} K_{2}\left(\eta^{\prime},-\tau\left(\eta^{\prime}\right)\right)=\left(\operatorname{grad} \tau\left(\eta^{\prime}\right), 1\right)$ for $a_{0}$ small enough.

Proof. We have

$$
\left(\operatorname{grad} K_{2}\right)\left(\eta^{\prime},-\tau\left(\eta^{\prime}\right)\right)=\left(\operatorname{grad} \tau\left(\eta^{\prime}\right), 1\right)\left(\partial K_{2} / \partial \eta_{n}\right)\left(\eta^{\prime},-\tau\left(\eta^{\prime}\right)\right)
$$

which yields $\left(\operatorname{grad} K_{2}\right)\left(\eta^{\prime},-\tau\left(\eta^{\prime}\right)\right) \in \Omega_{\gamma_{1}}^{\prime}$ since $\gamma_{1}<\gamma_{0}$ and

$$
\left|\partial K_{2} / \partial \eta_{n}-1\right| \leq C a^{N-3 / 2-(2 l+3) b} .
$$

Therefore

$$
\left(\operatorname{grad} K^{\prime}\right)\left(\eta^{\prime},-\tau\left(\eta^{\prime}\right)\right)=\left(\operatorname{grad} K_{2}\right)\left(\eta^{\prime},-\tau\left(\eta^{\prime}\right)\right) \in \Omega_{\gamma_{1}}^{\prime}
$$

and $\left(\partial K_{2} / \partial \eta_{n}\right)\left(\eta^{\prime},-\tau\left(\eta^{\prime}\right)\right)=1$ in view of (5.16) which proves the claim.

Now using (5.17), we obtain $\widetilde{T}\left(y^{\prime}, 0, \eta^{\prime},-\tau\left(\eta^{\prime}\right)\right) \in \mathrm{A}^{\prime}$ for any $\eta^{\prime} \in \mathbb{D}_{a, \gamma_{0}}^{n-1}$, $y^{\prime} \in \mathbb{T}^{n-1}$ where $A^{\prime}=\Sigma_{0} \cap\left\{y_{n}=0\right\}$ has the form

$$
\mathrm{A}^{\prime}=\left\{\left(y^{\prime}, 0, \eta^{\prime},-H^{0}\left(\eta^{\prime}\right)\right) ;\left(y^{\prime}, \eta^{\prime}\right) \in \mathrm{A}_{a}^{n-1}\right\} .
$$

Moreover, $F^{2 \pi}=\imath \mathrm{Bl}^{-1}: \mathrm{A}^{\prime} \rightarrow \mathrm{A}^{\prime}$ and from (5.18) and Lemma 5.5 we have

$$
{ }_{\imath} B l^{-1}\left(\tilde{T}\left(y^{\prime}, 0, \eta^{\prime},-\tau\left(\eta^{\prime}\right)\right)\right)=\tilde{T}\left(y^{\prime}+\operatorname{grad} \tau\left(\eta^{\prime}\right), 2 \pi, \eta^{\prime},-\tau\left(\eta^{\prime}\right)\right)
$$

for any $\eta^{\prime} \in \mathbb{D}_{a, \gamma_{0}}^{n-1}$ and $y \in \mathbb{T}^{n-1}$. 
Denote by $l_{1}$ the immersion

$$
l_{1}\left(y^{\prime}, \eta^{\prime}\right)=\left(y^{\prime}, 0, \eta^{\prime},-\tau\left(\eta^{\prime}\right)\right) \in \mathbb{T}^{n} \times \Sigma_{1}, \quad\left(y^{\prime}, \eta^{\prime}\right) \in \mathrm{A}_{a}^{n-1},
$$

and set $S\left(Y^{\prime}, \eta^{\prime}\right)=\tilde{S}\left(Y^{\prime}, 0, \eta^{\prime},-\tau\left(\eta^{\prime}\right)\right)$. This function satisfies (5.4) in view of (5.13) and (5.21). Denote by $U$ the symplectic map generated by $S$. Then $U$ maps $\mathrm{A}_{a}^{n-1}$ into a set of the same type. Moreover, $U\left(y^{\prime}, \eta^{\prime}\right)=l^{-1} \circ \widetilde{T} \circ l_{1}\left(y^{\prime}, \eta^{\prime}\right)$ for $\eta^{\prime} \in \mathbb{D}_{a, y_{0}}^{n-1}$ since $\left(\partial \widetilde{S} / \partial \eta_{n}\right)\left(Y, \eta^{\prime},-\tau\left(\eta^{\prime}\right)\right)=0$ for $\eta^{\prime} \in \mathbb{D}_{a, \gamma_{0}}^{n-1}$ in view of (5.17) and Lemma 5.5. Now (5.2) follows directly from (5.23). This completes the proof of Theorem 5.2.

Remark 5.1. Instead of $K_{2}(\eta)$ we can use any function $\widetilde{K}_{2}(\eta)=K_{2}(\eta)+g(\eta)$ such that $g(\eta)=0$ on $\mathbb{D}_{a, \eta_{1}}^{n}$ and

$$
\|g\|_{p, \gamma} \leq C_{p} a^{N-1 / 2-(l+1) b} \text { in } \mathbb{D}_{a}^{n} .
$$

Then the corresponding solution $\tilde{\tau}\left(\eta^{\prime}\right)$ of the equation $\widetilde{K}_{2}\left(\eta^{\prime}, \eta_{n}\right)=0$ with respect to $\eta_{n}$ equals $\tau\left(\eta^{\prime}\right)$ for any $\eta^{\prime} \in \mathbb{D}_{a, \gamma_{0}}^{n-1}$ and the sets $\mathbb{D}_{a, \gamma_{0}}^{n-1}$ and $\widetilde{\mathbb{D}}_{a, \gamma_{0}}^{n-1}$ defined by $\tau$ and $\tilde{\tau}$ coincide.

Indeed, writing down $\tilde{\tau}\left(\eta^{\prime}\right)=\tau\left(\eta^{\prime}\right)+r\left(\eta^{\prime}\right), K_{2}(\eta)=\left(\eta_{n}+\tau\left(\eta^{\prime}\right)\right) h(\eta)$ with some function $h(\eta) \neq 0$ in a neighbourhood of $\mathbb{D}_{a}^{n}$ and

$$
\widetilde{K}_{2}(\eta)=\left(\eta_{n}+\tau\left(\eta^{\prime}\right)+g_{1}(\eta)\right) h(\eta), \quad g_{1}(\eta)=g(\eta) / h(\eta),
$$

we have

$$
r\left(\eta^{\prime}\right)=g_{2}\left(\eta^{\prime}, r\left(\eta^{\prime}\right)\right), \quad g_{2}(\eta)=g_{1}\left(\eta^{\prime},-\tau\left(\eta^{\prime}\right)-\eta_{n}\right) .
$$

The function $g_{2}$ satisfies the estimate

$$
\left\|g_{2}\right\|_{p, \gamma_{2}} \leq C_{p} a^{N-1 / 2-(l+1) b}
$$

in view of (5.21) and (5.24). Then $\left(\eta^{\prime}, r\left(\eta^{\prime}\right)\right) \in \mathbb{D}_{a}^{n}$ for $\eta^{\prime} \in \mathbb{D}_{a}^{n-1}$ and by Lemma 5.5 we obtain the equality

$$
r\left(\eta^{\prime}\right)=g_{2}\left(\eta^{\prime}, r\left(\eta^{\prime}\right)\right)-g_{2}\left(\eta^{\prime}, 0\right)=r\left(\eta^{\prime}\right) v\left(\eta^{\prime}\right)
$$

for any $\eta^{\prime} \in \mathbb{D}_{a, \gamma_{0}}^{n-1}$. Here

$$
v\left(\eta^{\prime}\right)=\int_{0}^{1} \frac{\partial g_{2}}{\partial \eta_{n}}\left(\eta^{\prime}, s r\left(\eta^{\prime}\right)\right) d s=O\left(a^{N-3 / 2-(2 l+3) b}\right),
$$

thus $\left|v\left(\eta^{\prime}\right)\right|<1$ for $0<a \leq a_{0}$ and $a_{0}$ small enough. Now (5.26) implies $r\left(\eta^{\prime}\right)=0$ on $\mathbb{D}_{a, \gamma_{0}}^{n-1}$. Since $\mathbb{D}_{a, \gamma_{0}}^{n-1}$ has no isolated points, we have $\operatorname{grad} \tilde{\tau}\left(\eta^{\prime}\right)=$ $\operatorname{grad} \tau\left(\eta^{\prime}\right)$ for $\eta^{\prime} \in \mathbb{D}_{a, \gamma_{0}}^{n-1}$. On the other hand, the map $\mathbb{D}_{a}^{n-1} \ni \eta^{\prime} \rightarrow \operatorname{grad} \tilde{\tau}\left(\eta^{\prime}\right)$ is injective in view of Proposition 4.6 and (5.21) which proves the relation $\mathbb{D}_{a, \gamma_{0}}^{n-1}=\widetilde{\mathbb{D}}_{a, \gamma_{0}}^{n-1}$.

Proof of Theorem 1. Set $a_{j}=q^{j}, 0<q<a_{0}, \mathbb{D}_{j}=\mathbb{D}_{a_{j}}^{n-1}, \mathbf{A}_{j}=\mathbb{T}^{n-1} \times \mathbb{D}_{j}$, $\mathbb{D}_{j, \gamma}=\mathbb{D}_{a_{j}, \gamma}^{n-1}, \gamma_{j}^{0}=\gamma_{0}\left(a_{j}\right)=C_{5} a_{j}^{1+l b}$ and let $S_{j}(\theta, I)$ be the function $S(\theta, I)$ 
defined by Theorem 5.2 for $a=a_{j}$. Choose $\Gamma^{\prime}$ of the same type as $\Gamma, \Gamma \subset$ $\Gamma^{\prime} \subset \bigcup_{j=1}^{\infty} \mathbb{D}_{j}, \Gamma$ being defined by (2.4) with some constants $C_{k}^{0}, k=1, \ldots, 4$, while $\mathbb{D}_{j}$ are defined by (4.26) with some constants $C_{k}, k=1, \ldots, 4$. Denote by $\mathbb{D}_{j}^{\prime}$ the set (4.26) with constants $C_{k}^{0}$ and $a=a_{j}$. We can suppose that $\mathbb{D}_{j} \cap \mathbb{D}_{j+\nu}=\varnothing$ for any $j$ and $\nu \geq 2$ and that $\mathbb{D}_{j}^{\prime} \cap \mathbb{D}_{j+1}^{\prime}$ has a nonempty interior.

Proposition 5.6. There exist functions $K_{j}, S_{j}$ defined by Theorem 5.2 in $A_{j}$ with $\gamma_{j}^{0}=C_{5} a_{j}^{1+l b}$ and such that

$$
\begin{gathered}
K_{j}(I)=K_{j+1}(I) \quad \text { in } \mathbb{D}_{j} \cap \mathbb{D}_{j+1}, \\
S_{j}(\theta, I)=S_{j+1}(\theta, I)
\end{gathered}
$$

for any $I \in \mathbb{D}_{j, \gamma} \cap \mathbb{D}_{j+1}$ and $\gamma \geq \gamma_{j}^{0}$.

Proof. Denote by $\widetilde{T}_{j}$ the corresponding canonical transformation defined by Theorem 5.4. From Remark 1 after Pöschel's Theorem A [19] it follows that $\widetilde{T}_{j}^{-1} \circ \widetilde{T}_{j+1}(y, \eta)=(y+g(\eta), \eta)$ for $\eta \in \mathbb{D}_{a_{j}, \gamma_{j}^{1}}^{n} \cap \mathbb{D}_{a_{j}+1}^{n}$. Suppose that the exact symplectic map $T=\widetilde{T}_{j}^{-1} \circ \widetilde{T}_{j+1}$ is defined by the generating function $R(Y, \eta)$. Then $\operatorname{grad}_{Y} R(Y, \eta)$ does not depend on $Y \in \mathbb{T}^{n}$ for $\eta \in \mathbb{D}_{j, \gamma}^{n} \cap \mathbb{D}_{j+1}^{n}$, $\gamma \geq \gamma_{j}^{1}$ and since $R(Y, \eta)$ is small enough, we obtain $R(Y, \eta)=R(\eta)$. Thus $\widetilde{T}_{j+1}(y, \eta)=\widetilde{T}_{j}(y+\operatorname{grad} R(\eta), \eta), \eta \in \mathbb{D}_{j, \gamma}^{n} \cap \mathbb{D}_{j+1}^{n}$. Id) .

Moreover, the function $\widetilde{S}_{j}(Y, \eta)+R(\eta)$ generates the map $\widetilde{T}_{j} \circ(\mathrm{Id}+\operatorname{grad} R$,

Let $\varphi_{j}=\varphi_{j}(y, \eta)$ solve the equation

$$
\varphi-\frac{\partial \widetilde{S}_{j}}{\partial \eta}(\varphi, \eta)-\frac{\partial R}{\partial \eta}(\eta)=y
$$

and let $\varphi_{j+1}=\varphi_{j+1}(y, \eta)$ solve $\varphi-\partial \widetilde{S}_{j+1}(\varphi, \eta) / \partial \eta=y$.

Then

$$
\begin{aligned}
& \left(\varphi_{j+1}(y, \eta), \eta-\left(\partial \widetilde{S}_{j+1} / \partial Y\right)\left(\varphi_{j+1}(y, \eta), \eta\right)\right) \\
& \quad=\widetilde{T}_{j+1}(y, \eta)=\left(\varphi_{j}(y, \eta), \eta-\left(\partial \widetilde{S}_{j} / \partial Y\right)\left(\varphi_{j}(y, \eta), \eta\right)\right)
\end{aligned}
$$

and we have

$$
\varphi_{j+1}=\varphi_{j}, \quad \operatorname{grad}_{Y}\left(\widetilde{S}_{j+1}(Y, \eta)-\widetilde{S}(Y, \eta)\right)=0
$$

for $\eta \in \mathbb{D}_{j, \gamma}^{n} \cap \mathbb{D}_{j+1}^{n}$. As in the proof of Theorem 5.2 we have

$$
K_{1, j}(Y, \eta)=\widetilde{H}\left(Y, \eta-\left(\partial \widetilde{S}_{j} / \partial Y\right)(Y, \eta)\right)
$$

and

$$
K_{2, j}(\eta)=\widetilde{H}\left(0, \eta-\left(\partial \widetilde{S}_{j} / \partial Y\right)(0, \eta)\right) .
$$


In view of (5.29) we have $K_{2, j}(\eta)=K_{2, j+1}(\eta), \eta \in \mathbb{D}_{j, \gamma}^{n} \cap \mathbb{D}_{j+1}^{n}$. Now instead of $K_{2, j+1}(\eta)$ we consider $\widetilde{K}_{2, j+1}(\eta)=K_{2, j+1}(\eta)+g(\eta)$ where $g(\eta)=$ $K_{2, j}(\eta)-K_{2, j+1}(\eta)$ in $\mathbb{D}_{j}^{n} \cap \mathbb{D}_{j+1}^{n}, g(\eta)=0$ on $\mathbb{D}_{j+1, \gamma}^{n} \cup\left(\mathbb{D}_{j+1}^{n} \cap \mathbb{D}_{j+2}^{n}\right)$ and

$$
\|g\|_{p, \mathbb{D}_{j+1, \gamma}} \leq C_{p} a_{j+1}^{N-1 / 2-(l+1) b} .
$$

According to Remark 5.1 we can replace $K_{2, j+1}$ by $K_{2, j+1}+g$. Thus we can suppose that $K_{2, j+1}=K_{2, j}$ on $\mathbb{D}_{j}^{n} \cap \mathbb{D}_{j+1}^{n}$. Then $\tau_{j+1}(I)=\tau_{j}(I)$ in $\mathbb{D}_{j, \gamma} \cap \mathbb{D}_{j+1}$ and so in $\mathbb{D}_{j} \cap \mathbb{D}_{j+1}$. Therefore,

$$
S_{j+1}(\theta, I)=\widetilde{S}_{j+1}\left(\theta, 0, I,-\tau_{j+1}(I)\right)=\widetilde{S}_{j}\left(\theta, 0, I,-\tau_{j}(I)\right)=S_{j}(\theta, I)
$$

for $I \in \mathbb{D}_{j, \gamma} \cap \mathbb{D}_{j+1}, \gamma \geq \gamma_{j}^{1}$ and any $\theta \in \mathbb{T}^{n-1}$. This completes the proof of Proposition 5.6.

Remark 5.2. Making use of the representation (5.5) and approximating the smooth functions $Q_{\alpha}, Q^{\prime}$ with real-analytic ones, we can choose the sequence of analytic functions approximating the smooth Hamiltonian in the proof of Pöschel's Theorem B [19] independent of the domain. Thus equalities (5.27) and (5.28) may be assumed fulfilled by construction for any $I \in \mathbb{D}_{j} \cap \mathbb{D}_{j+1}$ and $\theta \in \mathbb{T}^{n-1}$.

Now we shall prove Theorem 1 . We can find smooth functions $\varphi_{j} \in C_{0}^{\infty}\left(\mathbb{D}_{j}\right)$ such that $\sum_{j=1}^{\infty} \varphi_{j}(I)=1$ for $I \in \Gamma^{\prime}$ and $\varphi_{j}=1$ on $\mathbb{D}_{j}^{\prime}$,

$$
\left|D^{\beta} \varphi_{j}\right| \leq C_{\beta} a_{j}^{-|\beta|}
$$

Denote $S(\theta, I)=\sum_{j=1}^{\infty} \varphi_{j}(I) S_{j}(\theta, I)$ and by $K(I)$ the smooth function $K$ such that $K(I)=K_{j}(I)$ in $\mathbb{D}_{j}, \tau(I)=-\frac{2}{3}(K(I))^{3 / 2}$. Then $S(\theta, I)=S_{j}(\theta, I)$ in $\mathbb{D}_{j, \gamma_{j}^{0}}$. For $\mu>0$ and $\sigma>n-1$ denote by $\Gamma_{\mu}$ the set

$$
\begin{aligned}
& \Gamma_{\mu}=\left\{I \in \Gamma ;\left|\langle\operatorname{grad} \tau(I), k\rangle-k_{n}\right| \geq \mu I_{1}^{1+l b}|k|^{-\sigma}\right. \\
&\text { for any } \left.k=\left(k^{\prime}, k_{n}\right) \in \mathbb{Z}^{n} \backslash\{0\}\right\},
\end{aligned}
$$

$E=\bar{\Gamma}_{\mu}=\Gamma_{\mu} \cup\left\{\left(0, t_{0}\right)\right\}$. We choose $C_{5}$ in the definition of $\gamma_{j}$ so that $\Gamma_{\mu} \cap$ $\mathbb{D}_{j} \subset \mathbb{D}_{j, y}$ for any $\gamma \leq \gamma_{j-1}^{0}$. This is satisfied if $\mu\left(C_{1} a_{j}\right)^{1+l b} \geq C_{5} a_{j-1}^{1+l b}$, i.e. $C_{5} \leq \mu\left(C_{1} q\right)^{1+l b}$. Then we have (5.30) and choosing $b<1 /(4 l+8)$ in an appropriate way, we can prove the desired smoothness of $K, g$ and $U$.

Now suppose that $N \geq 3$. From estimate (5.3) we obtain

$$
\left\|K_{j}-\zeta_{0}\right\|_{2, \mathbb{D}_{j}} \leq C_{2} a^{N-5 / 2-(3 l+7) b}
$$

and the right-hand side can be estimated by $C_{2} a^{b}$ if $b<1 /(6 l+16)$. In particular,

$$
\left\|\tau-H^{0}\right\|_{2, \mathbb{D}_{j}} \leq \widetilde{C}_{2} a^{b}
$$


Let $I \in \Gamma \backslash \Gamma_{\mu}$. Then $I \in \mathbb{D}_{j}$ for some $j$ and $\tau_{I}(I) \in \Omega_{a_{j}} \backslash \Omega_{a_{j}}^{\gamma_{j}}$ where $\gamma_{j}=\mu\left(C_{2} a_{j}\right)^{1+l b}$. Then by Lemma 5.1 we obtain

$$
\operatorname{mes}\left(\Omega_{a_{j}} \backslash \Omega_{a_{j}}^{\gamma_{j}}\right) \leq C \gamma_{j} a_{j}^{n-3+b} \leq C^{\prime} a_{j}^{n-2+(l+1) b}
$$

Then

$$
\begin{aligned}
\operatorname{mes}\left(\mathbb{D}_{j} \backslash \mathbb{D}_{j}^{\gamma_{j}}\right) & =\int_{\Omega_{a_{j}} \backslash \Omega_{a_{j}}^{\gamma_{j}}}\left|\operatorname{det} \tau_{I}^{-1}(\omega)\right| d \omega \\
& \leq \operatorname{mes}\left(\Omega_{a_{j}} \backslash \Omega_{a_{j}}^{\gamma_{j}}\right)\left(\min _{\mathbb{D}_{j}}\left|\operatorname{det} \tau_{I}(I)\right|\right)^{-1} \leq C a_{j}^{n-2+(l+4-n) b}
\end{aligned}
$$

in view of (4.29) and (5.31). Here and below we denote by $C, C^{\prime}$ various constants. Thus we obtain

$$
\begin{aligned}
\operatorname{mes}\left(\Gamma \backslash \Gamma_{\mu}\right) & \leq \sum_{j=1}^{\infty} \operatorname{mes}\left(\mathbb{D}_{j} \backslash \mathbb{D}_{j}^{\gamma_{j}}\right) \leq C \sum_{j=1}^{\infty} a_{j}^{n-2+(l+4-n) b} \\
& =C \sum_{j=1}^{\infty}\left(q^{j}\right)^{n-2+(l+4-n) b}<C^{\prime} q^{n-2+(l+4-n) b} .
\end{aligned}
$$

On the other hand,

$$
\operatorname{mes} \Gamma \geq C \sum_{j=1}^{\infty} a_{j}^{n-2+2 b}>C q^{n-2+2 b},
$$

hence $\operatorname{mes}\left(\Gamma \backslash \Gamma_{\mu}\right) / \operatorname{mes} \Gamma \leq C^{\prime} q^{(l+2-n) b} \leq C^{\prime} q^{b}$ provided that $l+2-n \geq 1$, i.e. $l \geq n-1$. This estimate immediately implies (2.6) and Theorem 1 is proved.

\section{The TWO-DIMENSIONAL CASE}

Proof of Theorem 2. Let $\zeta$ be an approximate interpolating Hamiltonian for the billiard ball map $B$ [14], i.e .

$$
B(\rho)=\exp \left(-\zeta^{1 / 2} X_{\zeta}\right)(\rho)+O\left(\zeta^{\infty}\right)
$$

in a neighbourhood of $S_{+}^{*} \partial \Omega$. Denote by $M_{r}$ the closed curve $M_{r}=\{\zeta=r\}$ for $r \in(-\delta, \delta), \delta$ small enough. For any $\rho \in M_{r}$ consider the map $\mathbb{R}^{1} \ni$ $t \rightarrow \exp \left(t X_{\zeta}\right)(\rho) \in M_{r}$ and denote by $l(r)$ its period. Let $S$ be a transversal to $M_{0}$. For $\rho \in M_{r}$ denote by $t(\rho)$ the smallest positive time $t$ such that $\exp \left(-t X_{\zeta}\right)(\rho) \in S$. Then $(t(\rho), \zeta(\rho)), 0 \leq t(\rho) \leq l(\zeta(\rho))$ are symplectic coordinates in a neighbourhood of $S_{+}^{*} \partial \Omega$. We define $\varphi(\rho)=2 \pi t(\rho) / l(\zeta(\rho))$ and complement it to exact symplectic coordinates $(\varphi, I) \in T^{*}\left(\mathbb{T}^{1}\right)$. For this purpose we seek for a function $I=g(\zeta)$ so that the change $\chi: T^{*} \partial \Omega \rightarrow T^{*}\left(\mathbb{T}^{1}\right)$ transforms the symplectic 1 -form $\sigma=\xi d s$ on $\Sigma$ into $I d \varphi$. Then $I$ and $\zeta$ satisfy the relation

$$
2 \pi I(\rho)=-\int_{0}^{\zeta(\rho)} l(r) d r+l_{0}
$$


$l_{0}=\int_{\gamma^{+}} \sigma=l(0)=2 \pi \mathfrak{l}$, where $\gamma^{+}$is $S_{+}^{*} \partial \Omega$ with the positive orientation. Denote by $\zeta(I)$ the solution of $(6.1)$ with respect to $\zeta$ with initial condition $\zeta(\mathfrak{l})=0$ and by $\zeta_{M}(I)$ its Taylor expansion up to degree $M$ which will be chosen large enough.

Now, in the coordinates $(\varphi, I)$ the exact symplectic map $B_{0}=\chi B \chi^{-1}$ can be written as

$$
B_{0}(\varphi, I)=\left(\varphi+\tau_{M}^{\prime}(I), I\right)+O\left((\mathfrak{l}-I)^{M+3 / 2}\right),
$$

$\tau_{M}=-\frac{2}{3} \zeta_{M}^{3 / 2}(I)$ in a neighbourhood of $\mathbb{T}^{1} \times\{\mathfrak{l}\}$. As above, we first consider $B_{0}$ in the sets

$$
\mathrm{A}_{j}=\mathbb{T}^{1} \times\left[\mathfrak{l}-C_{1} a_{j}, \mathfrak{l}-C_{2} a_{j}\right]=\mathbb{T}^{1} \times \Gamma_{j}, \quad j=1,2, \ldots,
$$

where $0<C_{2}<C_{1}, a_{j}=4^{-j}$ and $\mathrm{A}=\bigcup_{j=1}^{\infty} \mathrm{A}_{j}$. We choose $M_{j}=N+3 j / 2$, $N$ large enough, and consider $B_{0}$ in $A_{j}$ as the perturbation of the map $(\varphi, I) \rightarrow$ $\left(\varphi+\tau_{M_{j}}^{\prime}(I), I\right)$. By arguments similar to those in the proof of Theorem 5.2, replacing $l, b$ and $N$ by $j, 1 / 2$ and $N+(3 j+1) / 2$ respectively, we construct exact symplectic transformations $U_{j}: \mathrm{A}_{j} \rightarrow \mathrm{A}_{j}$ such that $B_{j}=U_{j}^{-1} B U_{j}$ are generated by the functions $\tau_{j}(I)+H_{j}(\varphi, I), j=1,2, \ldots, H_{j}(\varphi, I)=0$ for $(\varphi, I) \in \mathbb{T}^{1} \times E_{j}$ where $E_{j}$ are the preimages of the sets $R_{j}$ (see (2.8)) under $\tau_{j}^{\prime}$. Here for brevity we write $\tau_{j}$ instead of $\tau_{M_{j}}$. Now we choose $E=\bigcup_{j=1}^{\infty} E_{j}$. We have

$$
E_{j} \subset\left\{I \in \Gamma_{j} ;\left|\tau_{j}^{\prime} k_{1}-k_{2}\right| \geq \mu^{\prime}|I-\mathfrak{l}|^{1+j / 2}|k|^{-\sigma}, \forall k=\left(k_{1}, k_{2}\right) \in \mathbb{Z}^{2} \backslash\{0\}\right\}
$$

with some $\mu^{\prime}>0, \sigma>1$ and repeating some details of the proof of (2.6), we obtain (2.7).

We can glue $K_{j}, H_{j}$ and $U_{j}$ together using again Remark 1 after Pöschel's Theorem A [16] which in the case $n=2$ is quite obvious. Moreover,

$$
\left|\partial_{I}^{\beta}\left(K-\zeta_{M_{j}}\right)\right| \leq C_{\beta}|I-\mathfrak{l}|^{N-1-5 \beta / 2+j(1-\beta / 2)}, \quad I \in \Gamma_{j},
$$

thus the function $K$ is $C^{\infty}$ in $\Gamma$.

This completes the proof of Theorem 2 .

Denote $R=\left\{\omega(I)=\tau^{\prime}(I) ; I \in E\right\}$ and by $\omega \rightarrow I(\omega)$ the inverse map of the diffeomorphism $\left[\mathfrak{l}-\delta_{0}, \mathfrak{l}\right) \ni I \rightarrow \omega(I)$. Set $\Lambda_{\omega}=U\left(\mathbb{T}^{1} \times\{I(\omega)\}\right)$. Then $\Sigma_{R}=U\left(\mathbb{T}^{1} \times E\right)$ is the union of the invariant curves $\Lambda_{\omega}$ of $B$ with rotation numbers $\rho\left(\Lambda_{\omega}\right)=\omega \in R$. It obviously satisfies the inequality

$$
\operatorname{mes} \Sigma^{\delta}-\operatorname{mes}\left(\Sigma_{R} \cap \Sigma^{\delta}\right) \leq C_{N} \delta^{N}
$$

for any $N>0, \delta>0$ where $\Sigma^{\delta}=\partial \Omega \times[1-\delta, 1]$.

Corollary 6.1. There exists an approximate interpolating Hamiltonian $\zeta$ for the billiard ball map $B$ in a neighbourhood of $S_{+}^{*} \partial \Omega$ such that

$$
B(\rho)=\exp \left(-\zeta^{1 / 2} X_{\zeta}\right)(\rho)
$$

for any $\rho \in \Sigma_{R}$. 
Proof of Theorem 3. Let $\mathfrak{g} \in \Gamma(m, n), \mathfrak{g}=\left\{g_{1}, \ldots, g_{n}\right\}$, i.e. $B^{j}\left(g_{1}\right)=g_{1+j}$, $j=1, \ldots, n-1, B^{n}\left(g_{1}\right)=g_{1}$ and $\mathfrak{g}$ has a winding number $m$. Denote by $x_{j}$ the reflection points on $\partial \Omega$ corresponding to $g_{j}$ and set $g_{j}=\left(s_{j}, \sigma_{j}\right)$ where $s_{j}$ is the value of the natural parameter on $\partial \Omega$ at $x_{j}$ and $\sigma_{j}$ is the cosine of the angle between the oriented line segment linking $x_{j}$ with $x_{j+1}$ and the oriented tangent line at $x_{j}$. Let $d$ be the maximal length of the arcs linking $x_{j}$ with $x_{j+1}, j=1, \ldots, n$, and then we have $d \leq m l_{0} / n<\delta l_{0}$. Thus we obtain $0 \leq 1-\sigma_{j} \leq C(\delta), C(\delta)>0$ as $\delta>0$ which means that for $\delta>0$ small enough and $m / n<\delta$ we have $g_{j} \in U(\mathrm{~A}), 1 \leq j \leq n$, if $\mathfrak{g} \in \Gamma(m, n)$. From Birkhoff's theorem it follows that $\Gamma(m, n)=\varnothing$ for $n \geq n_{0}, n_{0}$ large enough.

We shall use the following lemma.

Lemma 6.2. Let $\left(m_{k}, n_{k}\right) \in \mathbb{N}^{2}, k=1,2, \ldots$ and $2 \pi m_{k} / n_{k} \rightarrow \omega$ as $k \rightarrow \infty$. Let $\mathfrak{g}^{k}-\left\{g_{1}^{k}, \ldots, g_{n_{k}}^{k}\right\} \in \Gamma\left(m_{k}, n_{k}\right)$ and $U^{-1}\left(g_{j}^{k}\right)=\left(\varphi_{j}^{k}, I_{k}^{k}\right)$ where the map $U$ is given by Theorem 2. Then the sequence $\left\{I_{j}^{k}, 1 \leq j \leq n_{k}, k=1,2, \ldots\right\}$ is convergent and tends to $I(\omega)$.

Proof. Denote by $Q_{1}(\varphi, I)$ the first component of the function $Q(\varphi, I)$ in Remark 2.2 and $p(\varphi, I)=\tau^{\prime}(I)+Q_{1}(\varphi, I)$. Then $p_{I}>0$ for $0<\mathfrak{l}-I<\varepsilon$, $\varepsilon>0$ small enough. Let $\mathfrak{g} \in \Gamma(m, n)$ and $U^{-1}\left(g_{j}\right)=\left(\varphi_{j}, I_{j}\right), g_{j} \in \mathfrak{g}$, $j=1, \ldots, n$, and let $I\left(\omega_{1}\right)<I_{1}<I\left(\omega_{2}\right), I\left(\omega_{i}\right) \in E, i=1,2$. Then $I\left(\omega_{1}\right)<I_{j}<I\left(\omega_{2}\right)$ for $j=1, \ldots, n$ and $\omega_{i} \in R, \omega_{1}<\omega_{2}$. Moreover, for the rotation numbers of $\Lambda_{\omega}$ and $\mathfrak{g}$ we have $\rho\left(\Lambda_{\omega_{i}}\right)=\tau^{\prime}\left(I\left(\omega_{i}\right)\right)=\omega_{i}$, $\rho(\mathfrak{g})=2 \pi \mathrm{m} / n$ and since $p$ is monotonely increasing with respect to $I$ for $\varphi$ fixed, we easily obtain $\omega_{1}<2 \pi m / n<\omega_{2}$.

Note that the set $R$ has no isolated points, thus if there exists a sequence $\mathfrak{g}^{k} \in \Gamma\left(m_{k}, n_{k}\right), \quad k=1,2, \ldots$ such that $I_{j_{k}}^{k} \notin(I(\omega)-\varepsilon, I(\omega)+\varepsilon)$, then $I_{j_{k}}^{k} \notin\left(I\left(\omega_{1}\right), I\left(\omega_{2}\right)\right)$ for any $k, j$ and some $I\left(\omega_{i}\right) \in E$ appropriately chosen, $I\left(\omega_{1}\right)<I(\omega)<I\left(\omega_{2}\right)$. Hence $2 \pi m_{k} / n_{k} \notin\left(\omega_{1}, \omega_{2}\right)$ which contradicts the condition of the lemma.

By Theorem $2 B_{0}$ is an exact symplectic map with a generating function $\tau(I)+G(\varphi, I)$. It is easy to see that for $\alpha_{0}=I d \varphi$ we have $B_{0}^{*} \alpha_{0}-\alpha_{0}=d f$ where $f(\varphi, I)=I \tau^{\prime}(I)-\tau(I)+h(\varphi, I)$ and $h(\varphi, I)=I G_{I}(\varphi, I)-G(\varphi, I)$ vanishes on $E$. Since the $L$-spectrum of $B$ is a symplectic invariant which coincides with the length spectrum of $\Omega$ (see [6]), we have

$$
L(\mathfrak{g})=\sum_{j=1}^{n} f\left(B_{0}^{j}\left(\varphi_{1}, I_{1}\right)\right)=\sum_{j=1}^{n} f\left(\varphi_{j}, I_{j}\right)
$$

where $L(\mathfrak{g})$ is the length of the periodic geodesic in $\Omega$ corresponding to $\mathfrak{g}=$ $\left\{g_{1}, \ldots, g_{n}\right\} \in \Gamma(m, n),\left(\varphi_{j}, I_{j}\right)=U^{-1}\left(g_{j}\right)$, provided that $m / n<\delta$ and $\delta$ is small enough.

Let $m_{k} / n_{k} \rightarrow \omega / 2 \pi$ and $\mathfrak{g}^{k} \in \Gamma\left(m_{k}, n_{k}\right)$. From Lemma 6.2 we have $\mid I_{j}^{k}-$ $I(\omega) \mid \leq \varepsilon$ for $k \geq k(\varepsilon)>0$ where $\varepsilon=\varepsilon(\omega)$ is chosen so that $0<\varepsilon<$ 
$|I(\omega)-\mathfrak{l}| / 2$. Then $\tau(I)=-\frac{2}{3}(K(I))^{3 / 2}$ is a function of class $C^{\infty}$ in the interval $|I-I(\omega)|<\varepsilon$ and

$$
\left|L\left(\mathfrak{g}^{k}\right) / n_{k}-(\omega I(\omega)-\tau(I(\omega)))\right| \leq C(\omega) \max \left|I_{j}^{k}-I(\omega)\right| .
$$

Hence the sequence $L\left(\mathfrak{g}^{k}\right) / n_{k}, k=1,2, \ldots$, is convergent and for any choice of $\mathfrak{g}^{k} \in \Gamma\left(m_{k}, n_{k}\right)$ we have

$$
\lim _{k \rightarrow \infty} L\left(\mathfrak{g}^{k}\right) / n_{k}=S(\omega)
$$

where $S(\omega)=\omega I(\omega)-\tau(I(\omega))$ is just the Legendre transform of $\tau(I)$. Thus $L(m, n), m / n \leq \delta$, recover uniquely the Legendre transform of $\tau(I)$ in $R$. Since $E$ has no isolated points, one can recover from (6.2) the function $\tau(I)$ for any $I \in E$.

Now let $\Omega_{1}$ and $\Omega_{2}$ be two strictly convex domains in $\mathbb{R}^{2}, \Sigma^{i}=B^{*} \partial \Omega_{i}$; let $B_{i}$ be the respective billiard ball maps, $i=1,2$, and let the assumptions of Theorem 3 be satisfied. Let $E_{i}$ be defined by $E_{i}=\left\{I \in\left(\mathfrak{l}_{i}-\delta, \mathfrak{l}_{i}\right] ; \tau_{i}^{\prime}(I) \in R\right\}$, $\mathfrak{l}_{i}=l_{0}^{i} / 2 \pi, i=1,2$. It is easily seen that $l_{0}^{1}=l_{0}^{2}$ since $l_{0}^{i}=\lim _{n \rightarrow \infty} L\left(\mathfrak{g}^{n, i}\right)$, $\mathfrak{g}^{n, i} \in \Gamma^{i}(1, n)$.

Now for all $\omega \in R$ we have

$$
S_{1}(\omega)=S_{2}(\omega), \quad S_{i}(\omega)=\omega I_{i}(\omega)-\tau_{i}\left(I_{i}(\omega)\right), \quad i=1,2 .
$$

Since $R$ has no isolated points, we can differentiate with respect to $\omega$ which yields $I_{1}(\omega)=I_{2}(\omega)$. Hence $E_{1}=E_{2}$ and $\tau_{1}(I)=\tau_{2}(I)$ for $I \in E_{1}$, thus $B_{0,1}(\varphi, I)=B_{0,2}(\varphi, I)$ for any $(\varphi, I) \in \mathbb{T}^{1} \times E_{1}$. Conjugating with $U_{i}, i=1$, 2 , we obtain the assertion of Theorem 3 .

\section{CONCLUDING REMARKS}

7.1. A question may arise whether there exist strictly convex hypersurfaces with closed elliptic geodesics satisfying conditions (2.1) and (2.3). We can answer this question positively at least in the case $n=3$, perturbing arbitrarily little the metric on an ellipsoid with three different axes in an arbitrarily small neighbourhood of any point of the shortest or the longest ellipse and applying a classical result of Nirenberg (see [7]) on the isometrical embeddings of compact oriented surfaces with Riemannian metric with strictly positive curvature as smooth convex surfaces in $\mathbb{R}^{3}$.

7.2. Theorem 1 and a theorem of Birkhoff-Lewis type (cf. [16]) show that the closure of the periodic points of $B$ in a neighbourhood of $\widetilde{\mathscr{Q}}$ has a positive measure.

7.3. The results obtained hold also for any generalised billiard ball map arising as a boundary map for a pair of glancing hypersurfaces (see [15]). Indeed, 
we used in the proof only some properties of the approximated interpolating Hamiltonian but not the specific structure of $\Sigma$.

\section{APPENDIX}

As noted in $\S 5$, Theorem 5.4 can be derived from Pöschel's Theorem A [19]. The constants participating in the estimates throughout [19] depend only on $n$, $\sigma, \lambda, \rho, R$ and $\alpha$, but not on the domain or the parameter $\gamma$, i.e. their dependence on $R$, and, in Theorem $\mathrm{A}$, on $\rho$, is not stated explicitly. In our case we have $R=C a^{-b}, 0<\rho \leq a / 2$, the parameters $a$ and $b$ participating in the definitions of the domain $\mathbb{D}_{a}^{n}$ and the parameter $\gamma$ as well, thus we have to follow the dependence of the various constants in [19] on $R$ and, in Theorem A, on $\rho$ as well.

The Main Lemma in [19] holds for $\delta>0$ small enough depending only on $n, \sigma, \lambda, R$ and $\alpha$, but not on the domain, $\gamma$ or $\rho$. We put $\delta \leq C_{1} R^{-3}$ and follow the dependence of the constants $c_{2}, c_{3}, c_{8}, c_{11}$ in estimates (i), (ii), (iii), (v) on $R$.

In the proof of the Main Lemma a version of the implicit function theorem is used to solve for a local diffeomorphism $\varphi$ and a small perturbation $\hat{\varphi}$ the equation

$$
\varphi(\pi(\zeta))=\varphi(\zeta)+\hat{\varphi}(\zeta)
$$

for a map $\pi$ close to the identity $I$ :

Lemma A.1 [19, Lemma 4.2]. Let $\varphi, \hat{\varphi}$ be real analytic on the $\rho$-neighbourhood $\Lambda+\rho$ of a domain $\Lambda$ in $\mathbb{C}^{n}$, with $|D \varphi|,\left|D \varphi^{-1}\right| \leq S$ there. If

$$
|\hat{\varphi}|_{\Lambda+\rho} \leq \rho / c
$$

with a constant $c=c(n, S)$, then there exists a unique real analytic map

$$
\pi: \Lambda \rightarrow \Lambda+\rho, \quad|\pi-I|_{\Lambda} \leq 2 S|\hat{\varphi}|_{\Lambda+\rho}
$$

such that (A.1) holds. In addition, analytic dependence on parameters carries over from $\varphi, \hat{\varphi}$ to $\pi$. Also, $\pi-I$ is $2 \pi$-periodic in $\zeta$, if $\varphi$ and $\hat{\varphi}$ are.

Following the proof of the above lemma, we see that we can choose the constant $c=c(n, S)$ in (A.2) in the form $c=c(n)\left(1+S^{3}\right)$. Further on, for any $\delta \leq C_{1} R^{-3}, R$ large enough, the Main Lemma holds and the constants $c_{2}, c_{3}, c_{11}$ can be chosen independent of $R$ while $c_{8}$ depends linearly on $R$.

From the Main Lemma we can derive Theorem B for the normalized value of $\gamma=1 \leq \rho$. We find that the constant $c_{12}$ in [19, estimate (4.20)] depends linearly on $R$ while the constant $c_{13}$ in $[19,(4.23)]$ can be chosen independent of $R$. This provides an additional factor $R^{-1}$ in the left-hand side of the first estimate (3.24) in [19] for $\gamma=1$ while the second estimate (3.24) and (3.25) in [19] for $\gamma=1$ remain unchanged, with constant $c_{\beta}$ independent of $R$.

Now we pass from the normalized value of $\gamma=1 \leq \rho$ to the general case $0<\gamma \leq \rho$. We replace the functions $F$ and $G$ in the formulation of Theorem 
B in [19] by $\widetilde{F}^{0}=\gamma^{-2} F^{0} \circ \sigma_{\gamma}, \widetilde{G}=\gamma^{-2} G \circ \sigma_{\gamma}$ respectively and apply Theorem B for $\gamma=1$ and the same $R$ to the functions $\widetilde{F}^{0}$ and $\widetilde{G}$. This gives us functions $\widetilde{\Phi}, \widetilde{F}$, and $\widetilde{\Gamma}$ satisfying equalities (3.22), (3.23) in [19] for $\widetilde{F}^{0}, \widetilde{G}$ instead of $F^{0}, G$ as well as estimates (3.24), (3.25) in [19] for $\gamma=1$, the left-hand side of the first inequality of (3.24) being multiplied by $R^{-1}$ and the constant $c_{\beta}$ independent of $R$. Now, the functions $\Phi=\sigma_{\gamma} \circ \widetilde{\Phi} \circ \sigma_{\gamma}^{-1}, F=\gamma^{2} \widetilde{F} \circ \sigma_{\gamma}^{-1}$ and $\Gamma=\gamma \tilde{\Gamma} \circ \sigma_{\gamma}^{-1}$ satisfy (3.22), (3.23) in [19] with the functions $F^{0}, G$. Moreover, estimates (3.24) in [19] hold for any $\gamma \leq \rho$, the left-hand side of the first estimate (3.24) being multiplied by $R^{-1}$. On the other hand, we see that the exponent of $\gamma$ in the right-hand side of (3.25) in [19] must be -1 and not -2 .

Now let the assumptions of Theorem $A$ be satisfied, the left-hand side of inequality (3.3) in [19] being multiplied by $R$ and $\delta \leq C_{1} R^{-3}, C_{1}$ independent of $R$ and $\rho$, i.e. the assumptions of Theorem 5.4 hold. Then from Theorem B [19] we find, on account of the correction in estimate (3.25), that instead of (3.7) in [19] the generating function $S$ satisfies the estimate

$$
\|S\|_{\widetilde{\beta} \lambda, \tilde{\beta} ; \gamma} \leq C_{\beta} \gamma^{-1} R^{\beta+1}\left\|H-H^{0}\right\|_{\beta \lambda+\lambda+\sigma ; \gamma}
$$

$C_{\beta}$ independent of $R$ and $\rho$, which corresponds to estimate (ii) in Theorem 5.4. Moreover, Theorem A provides a diffeomorphism $T: \mathbb{T}^{n} \times \Omega \rightarrow \mathbb{T}^{n} \times$ $I$ transforming the Hamiltonian vector field $X_{H}$ on $\mathbb{T}^{n} \times I$ into the vector field $T^{*} X_{H}$ on $T^{n} \times \Omega$ so that $\left.T^{*} X_{H}\right|_{T^{n} \times \Omega}=\langle\omega, \partial / \partial \theta\rangle$. Now, in order to obtain Theorem 5.4, we replace $S, \gamma, H, H_{0}$ and $I$ by $\widetilde{S}, \gamma_{1}, H^{\prime}, H_{0}^{\prime}$ and $\mathbb{D}_{a}^{n}$, respectively, and the canonical map $\widetilde{T}$ generated by $\widetilde{S}$ satisfies for $(y, \eta) \in \mathrm{A}_{a, \gamma_{1}}^{n}$ the equality $\widetilde{T}(y, \eta)=T\left(y,\left(\partial K^{\prime} / \partial \eta\right)(\eta)\right)$ where $K^{\prime}(\eta)$ is the nondegenerate Hamiltonian in (i) of Theorem $5.4(K(P)$ in $[19,(3.5)])$.

\section{REFERENCES}

1. V. I. Arnold, Small denominators and problems of stability of motion in classical and celestial mechanics, Russian Math. Surveys 18 (1963), 85-193.

2. __ Mathematical methods of classical mechanics, Springer-Verlag, Berlin and New York, 1978.

3. V. I. Arnold, V. V. Kozlov and A. I. Neustadt, Mathematical aspects of classical and celestial mechanics, Current Problems in Math., Fundamental Directions 3, Moscow, 1985. (Russian)

4. R. Douady, Une démonstration directe de l'équivalence des théorèmes de tores invariants pour difféomorphismes et champs de vecteurs, C. R. Acad. Sci. Paris Sér. A 295 (1982), 201-204.

5. __ Applications du theorème de tores invariants, Thèse, Univ. Paris VII, 1982.

6. V. Guillemin and R. Melrose, A cohomological invariant of discrete dynamical systems, Christoffel Centennial Volume, Birkhäuser, Basel, 1981.

7. R. Hamilton, The inverse function theorem of Nash and Moser, Bull. Amer. Math. Soc. 7 (1982), 65-222.

8. L. Hörmander, The analysis of linear partial differential operators. III, Springer-Verlag, Berlin and New York, 1985. 
9. W. Klingenberg, Lectures on closed geodesics, Springer-Verlag, Berlin and New York, 1978.

10. __ Riemannian geometry, de Gruyter, Berlin and New York, 1982.

11. V. Kovachev and G. Popov, Existence of invariant tori for the billiard ball map near an elliptic periodic geodesic, C. R. Acad. Bulgare Sci. 41 (1988), 19-22.

12. V. F. Lazutkin, Convex billiard and eigenfunctions of the Laplace operator, Leningrad Univ., 1981. (Russian)

13. A. Magnuson, Symplectic singularities, periodic orbits of the billiard ball map, and the obstacle problem, Thesis, M.I.T., Cambridge, Mass., 1984.

14. Sh. Marvizi and R. Melrose, Spectral invariants of convex planar regions, J. Differential Geom. 17 (1982), 475-502.

15. R. Melrose, Equivalence of glancing hypersurfaces, Invent. Math. 37 (1976), 165-191.

16. J. Moser, Proof of a generalized form of a fixed point theorem due to G. D. Birkhoff, Lecture Notes in Math., vol. 597, Springer-Verlag, Berlin and New York, 1977, pp. 464-494.

17. G. Popov, Invariant circles and length spectrum of the billiard ball map, Preprint.

18. __ Quasimodes for the Laplace operator (in preparation).

19. J. Pöschel, Integrability of Hamiltonian systems on Cantor sets, Comm. Pure Appl. Math. 35 (1982), 653-696.

20. N. V. Svanidze, Existence of invariant tori for a three-dimensional billiard, which are concentrated in the vicinity of a "closed geodesic on the boundary region," Uspekhi Mat. Nauk 33 (1978), 225-226. (Russian)

Institute of Mathematics, Bulgarian Academy of Sciences, Acad. G. Bonchev Street 8, Sofia 1113, Bulgaria 